\title{
Search for Leptoquarks Coupled to Third-Generation Quarks in Proton-Proton Collisions at $\sqrt{s}=13 \mathrm{TeV}$
}

\author{
A. M. Sirunyan et al. \\ (CMS Collaboration)
}

(Received 14 September 2018; published 12 December 2018)

\begin{abstract}
Three of the most significant measured deviations from standard model predictions, the enhanced decay rate for $B \rightarrow D^{(*)} \tau \nu$, hints of lepton universality violation in $B \rightarrow K^{(*)} \ell \ell$ decays, and the anomalous magnetic moment of the muon, can be explained by the existence of leptoquarks (LQs) with large couplings to third-generation quarks and masses at the $\mathrm{TeV}$ scale. The existence of these states can be probed at the LHC in high energy proton-proton collisions. A novel search is presented for pair production of LQs coupled to a top quark and a muon using data at a center-of-mass energy of $13 \mathrm{TeV}$, corresponding to an integrated luminosity of $35.9 \mathrm{fb}^{-1}$, recorded by the CMS experiment. No deviation from the standard model prediction has been observed and scalar LQs decaying exclusively into $t \mu$ are excluded up to masses of $1420 \mathrm{GeV}$. The results of this search are combined with those from previous searches for LQ decays into $t \tau$ and $b \nu$, which excluded scalar LQs below masses of 900 and $1080 \mathrm{GeV}$. Vector LQs are excluded up to masses of $1190 \mathrm{GeV}$ for all possible combinations of branching fractions to $t \mu, t \tau$ and $b \nu$. With this analysis, all relevant couplings of LQs with an electric charge of $-1 / 3$ to third-generation quarks are probed for the first time.
\end{abstract}

DOI: 10.1103/PhysRevLett.121.241802

The standard model of particle physics has been outstandingly successful in describing most fundamental physical phenomena. However, significant deviations from the predictions of the standard model (SM) have been observed in measurements of rare decays of $B$ mesons. In particular, deviations have been seen in the values of the ratio $R_{D^{(*)}}$, defined as the ratio of the $B \rightarrow D^{(*)} \tau \nu$ branching fraction to the $B \rightarrow D^{(*)} \mu \nu$ branching fraction. These deviations from the SM were first reported by the BABAR [1,2] and Belle [3-5] Collaborations and have been confirmed by the LHCb Collaboration [6,7] with a combined significance of about four standard deviations [8]. The ratios of the branching fractions of $B \rightarrow K^{(*)} \mu \mu$ to $B \rightarrow K^{(*)} e e, R_{K}$ and $R_{K^{*}}$, as measured by the LHCb Collaboration [9-12], show departures from lepton universality by 2.6 and 2.4 standard deviations, respectively. The measurement of the muon anomalous magnetic moment $a_{\mu}$, one of the most precisely measured quantities in particle physics [13], also deviates from the SM prediction by 3.5 standard deviations [14]. These anomalies are among the most significant deviations from the SM observed so far.

\footnotetext{
*Full author list given at the end of the Letter.

Published by the American Physical Society under the terms of the Creative Commons Attribution 4.0 International license. Further distribution of this work must maintain attribution to the author(s) and the published article's title, journal citation, and DOI. Funded by SCOAP ${ }^{3}$.
}

The existence of leptoquarks (LQs) with masses at the $\mathrm{TeV}$ scale and large couplings to third-generation quarks [15-25] has been proposed as a possible explanation for one, two, or all of these deviations. Leptoquarks are hypothetical particles that can decay to SM quarks and leptons. They are triplets with respect to the strong interaction, have fractional electric charge, and can be either scalar (spin 0) or vector (spin 1) particles. Many extensions to the SM, among them grand unification [26-28], technicolor [29,30], and compositeness models [31,32], predict the existence of these particles. The effective Buchmüller-Rückl-Wyler model [33] incorporates the assumption that LQ interactions with SM fermions are renormalizable and gauge invariant, leading to restrictions on the allowed quantum numbers of LQs [34]. Depending on its quantum numbers and the coupling structure, a given LQ can decay to any one of a number of different combinations of SM fermions. The couplings of LQs to leptons and quarks of different generations introduce flavor changing neutral currents that may be observable in precision measurements [35]. While simultaneous couplings to the first and second generations are tightly constrained by experimental data, the bounds are weaker for couplings to the second and third generation, thus allowing the existence of leptoquarks with nondiagonal couplings in the generation matrix $[19,24,36]$.

Collider searches for LQs with decays to thirdgeneration quarks have been performed in the decay channels LQ $\rightarrow t \tau, \mathrm{LQ} \rightarrow b \tau$, and LQ $\rightarrow b \nu$ at $\sqrt{s}=8 \mathrm{TeV}$ [37-44] and recently at $\sqrt{s}=13 \mathrm{TeV}$ [45-49]. We present 
the first search for the pair production of LQs with decays to a top quark and a muon, $\mathrm{LQ} \rightarrow t \mu$, a decay mode that is essential to explain the anomalies in $a_{\mu}$ and $R_{K^{(*)}}$ [19-25]. This search is combined with previous searches that target other decay modes $[48,49]$. The combination provides sensitivity to all relevant couplings of LQs with an electric charge of $-1 / 3$ to third-generation quarks.

At the CERN LHC, pair production of LQs is possible via gluon-gluon fusion or quark-antiquark annihilation, allowing direct searches to be performed. Single LQ production via quark-gluon scattering is subdominant for LQs coupled to heavy quarks, as it requires a heavy quark in the initial state. The pair production cross section depends on the mass of the scalar LQ and is known at next-to-leading order (NLO) precision [50]. The pair production cross section for vector LQs has been calculated at leading order (LO) [51] and is much larger than the scalar LQ cross section. The cross section for vector LQs depends on an additional parameter $\kappa$, which is a dimensionless coupling and takes a value of $\kappa=1$ in the Yang-Mills case and $\kappa=0$ in the minimal coupling case.

The central feature of the CMS apparatus is a superconducting solenoid of $6 \mathrm{~m}$ internal diameter, providing a magnetic field of $3.8 \mathrm{~T}$. Within the solenoid volume are a silicon pixel and strip tracker, a lead tungstate crystal electromagnetic calorimeter, and a brass and scintillator hadron calorimeter, each composed of a barrel and two endcap sections. Forward calorimeters extend the pseudorapidity $(\eta)$ coverage provided by the barrel and endcap detectors. Muons are detected in gas-ionization chambers embedded in the steel flux-return yoke outside the solenoid. A more detailed description of the CMS detector, together with a definition of the coordinate system used and the relevant kinematic variables, can be found in Ref. [52].

This analysis uses data recorded by the CMS detector in $p p$ collisions at a center-of-mass energy of $13 \mathrm{TeV}$ in 2016. Online, potential signal events are required to pass a singlemuon trigger that selects isolated muon candidates with transverse momentum $p_{T}>24 \mathrm{GeV}$ [53]. Data recorded by single electron triggers are used in background-enriched control regions (CRs). The data correspond to an integrated luminosity of $35.9 \mathrm{fb}^{-1}$.

Signal events of pair-produced LQs with prompt decays to $t \mu$ are simulated with the PYTHIA 8.205 [54,55] Monte Carlo program at $\mathrm{LO}$ for mass values ranging from 200 to $2000 \mathrm{GeV}$. The POWHEG [56-63] v1 generator is used to simulate background events resulting from the production of single top quarks in the $t W$ channel at NLO. The POWHEG v2 generator is used for single top production in the $t$ channel and for simulating $t \bar{t}$ production at NLO. Single top quark production in the $s$ channel, $t \bar{t}$ production in association with a heavy gauge boson $(t \bar{t}+\mathrm{V})$, and the production of a $W$ boson with additional jet radiation are simulated with MADGRAPH 5_aMC@NLO (v2.2.2) [64] at NLO. Events from Drell-Yan (DY) production with additional jet radiation are simulated with MADGRAPH 5_aMC@NLO at LO and an NLO $K$ factor is applied to the LO DY + jets production cross section. The simulation of the production of two heavy gauge bosons with additional jet radiation is performed at NLO with MADGRAPH 5_aMC@NLO and POWHEG v2. Events in which jets are produced through the strong interaction only, referred to as quantum chromodynamic multijet events, are simulated with PYTHIA at LO.

Parton showers in the simulated $W$ boson production events and DY events with additional jet radiation are matched to the matrix element calculation with the FXFX [65] and MLM [66] algorithms, respectively. The parton shower and hadronization process is simulated with PYTHIA. The NNPDF3.0 [67] parton distribution functions (PDFs) at LO and NLO are used for processes simulated at LO and NLO, respectively. The underlying event tune CUETP8M2T4 [68] is used for the simulation of $t \bar{t}$ and single top quark production via the $t$ channel, all other processes are generated using CUETP8M1 [69,70]. All simulated event samples include the simulation of additional inelastic $p p$ interactions within the same or adjacent bunch crossings (pileup). The detector response is simulated with the GEANT4 package [71,72]. Simulated events are processed through the software chain used for collision data and are reweighted to match the observed distribution of the number of pileup interactions in data.

The CMS experiment uses a particle-flow (PF) event reconstruction algorithm [73], which makes use of an optimized combination of information from the various elements of the CMS detector. The reconstructed vertex with the largest value of summed physics object $p_{T}^{2}$ is taken to be the primary $p p$ interaction vertex. The physics objects here are the objects returned by a jet finding algorithm $[74,75]$ applied to all charged tracks associated with the vertex, plus the associated missing transverse momentum, taken as the negative vector $p_{T}$ sum of those jets. More details are given in Ref. [76]. All detected particles are reconstructed either as electrons, muons, photons, charged hadrons, or neutral hadrons. In this analysis, electrons and muons are required to have $p_{T} \geq 30 \mathrm{GeV},|\eta| \leq 2.4$, and to be isolated. The isolation $[77,78]$ is defined as the summed $p_{T}$ of all neutral particles and charged hadrons in a cone with radius $\Delta R=\sqrt{(\Delta \eta)^{2}+(\Delta \phi)^{2}}$, $\phi$ being the azimuthal angle in radians, of 0.4 (for muons) or 0.3 (for electrons) around the lepton. The sum is corrected for the contribution of neutral pileup inside the cone. Jets are clustered from charged and neutral PF candidates using the anti- $k_{T}$ jetclustering algorithm [74,75] with a distance parameter of 0.4 . Charged PF candidates originating from vertices other than the primary vertex are not clustered. A jet energy correction (JEC) is applied [79] to account for remaining contributions arising from a different vertex than the primary one as well as for nonuniformity of the jet response in $\eta$ and nonlinearity in $p_{T}$. Finally, a correction is applied 
to account for the residual differences in the jet response between data and simulated events. The jet energy resolution (JER) in simulated events is smeared to match the wider resolution in data. All jets are required to have $p_{T} \geq 30 \mathrm{GeV}$ and $|\eta| \leq 2.4$. The combined secondary vertex v2 [80] algorithm is used to identify jets originating from bottom quarks ( $b$-tagged jets). The loose working point is chosen, which has an efficiency of about $90 \%$ and a mistag rate of approximately $10 \%$. The missing transverse momentum $p_{T}^{\text {miss }}$ is calculated as the magnitude of the negative vectorial $p_{T}$ sum of all PF candidates in an event. Both the jet energy scale and resolution corrections are propagated to the calculation of $p_{T}^{\text {miss }}$

Offline, events are required to contain at least two muons and at least two jets, of which at least one must be $b$ tagged. By requiring the invariant mass of each pair of muons in an event to exceed the $Z$ boson mass by at least $20 \mathrm{GeV}$, events arising from the production of a $Z$ boson with additional jet radiation are suppressed. As the decay of heavy LQs is expected to produce highly energetic leptons and jets, minimum values of $S_{T}^{\text {lep }}$ and $S_{T}$ of 200 and $350 \mathrm{GeV}$ are required, respectively. Here, $S_{T}^{\text {lep }}$ is the scalar $p_{T}$ sum of all selected muons and electrons and $S_{T}$ is defined as the scalar sum of $S_{T}^{\text {lep }}, p_{T}^{\text {miss }}$, and the $p_{T}$ of all selected jets. The phase space region resulting from these selection criteria is referred to as the signal region (SR) in the following.

Two categories of events are defined, based on the number of muons or electrons. If at least three such charged leptons are present, of which at least two are muons, and at least one pair of muons with opposite electric charge is found, the event falls into category $A$. Category $B$ contains all remaining events in the SR. In category $A$, the LQ mass $M_{\mathrm{LQ}}^{\mathrm{rec}}$ is reconstructed under the assumption that one of the top quarks decays into the leptonic final state with a muon or an electron (leptonic top) and the other one decays into the hadronic final state (hadronic top). The distribution of $M_{\mathrm{LQ}}^{\mathrm{rec}}$ is used for the final statistical analysis in this category, while the distribution of $S_{T}$ is used for this purpose in category $B$.

For each event, the leptonic top quark candidates are constructed from permutations of one or more of the seven $p_{T}$-leading jets, one of the three $p_{T}$-leading muons or the $p_{T}$-leading electron, and $p_{T}^{\text {miss }}$. The hadronic top quark candidates are constructed using all permutations of jets not assigned to the leptonic top quark. The LQ candidates are assembled from top quark candidates and the two $p_{T^{-}}$ leading muons that have not been associated to the leptonic top quark. The muon charge is used when assigning it to one of the top quark candidates. In events with more than two muons, all possible permutations of muons are considered. A $\chi^{2}$ variable that takes into account the invariant mass of each top quark candidate and the relative mass difference between the two LQ candidates is then used to select the best pair of LQ candidates for each event. Events with four leptons, which could originate from dileptonic $t \bar{t}$ decays, are included in category $A$ and contribute to the signal efficiency. In order to provide a more accurate SM background prediction in category $A$, which contains a minimum of three charged leptons, the misidentification rate of electrons and muons is measured using jets in a $\mathrm{DY}+$ jets enriched $\mathrm{CR}$ in data. The $\mathrm{CR}$ is defined by selecting two muons with an invariant mass close to the $Z$ boson mass, and the misidentification rate is measured on events where a jet is misidentified as a third lepton. The resulting data-to-simulation correction factors are applied to simulation for each misidentified charged lepton in a given event in the SR. The effect of charge misidentification on the analysis was found to be negligibly small.

The contributions from the dominant SM backgrounds in category $B$, the production of $t \bar{t}$ and DY + jets events, are estimated simultaneously in a data-driven procedure. A CR similar to the SR is defined by requiring a minimum of two electrons without additional muons. The invariant mass of any pair of electrons must be at least $20 \mathrm{GeV}$ above the $Z$ boson mass and all other SR requirements have to be fulfilled for the selected events. We correct for small differences in the distribution of $S_{T}$ between the SR and the CR with an extrapolation function $\alpha\left(S_{T}\right)$, which is derived from simulated $t \bar{t}$ and $\mathrm{DY}+$ jets events by fitting both $S_{T}$ distributions with an empirical functional form to obtain smoothed distributions that are then used to compute the ratio. The number of data events in the CR, after all simulated minor backgrounds have been subtracted, is multiplied by $\alpha\left(S_{T}\right)$ to extrapolate into the SR. Using the ratio of the fitted functions results in a significantly smaller impact of systematic uncertainties on the estimated backgrounds.

Various uncertainties affecting the rate and the shape of the signal and background contributions are taken into account. In general, uncertainties in this analysis are treated similarly to those in Ref. [48]. For the background in category $A$, the uncertainties in the renormalization and factorization scales as well as the uncertainties in the lepton misidentification rates are dominant. In category $B$ the major backgrounds are derived from data. Lepton efficiencies and the background extrapolation procedure are the most important sources of uncertainties for these backgrounds. Uncertainties in the renormalization and factorization scales and in those associated with the choice of PDFs $[67,81]$ used to simulate the events dominate for the minor backgrounds. The signal in both categories is most affected by the uncertainties in lepton and $b$-tagging [80] efficiencies. Other uncertainties considered are related to SM cross sections [82-90], the integrated luminosity [91], JEC and JER [79], and the pileup reweighting [92].

The THETA software package [93] is used to perform a maximum-likelihood template fit to the binned $M_{\mathrm{LQ}}^{\mathrm{rec}}$ and $S_{T}$ distributions for the background and to extract the cross section of a potential signal. The statistical uncertainties in 
the SM backgrounds and the signal, as well as all systematic uncertainties, are taken into account as nuisance parameters in the fit. The uncertainty in the luminosity is assigned a log-normal prior distribution, for all other systematic uncertainties a Gaussian prior is used. The statistical uncertainty in the predicted background and the signal is taken into account by defining one additional nuisance
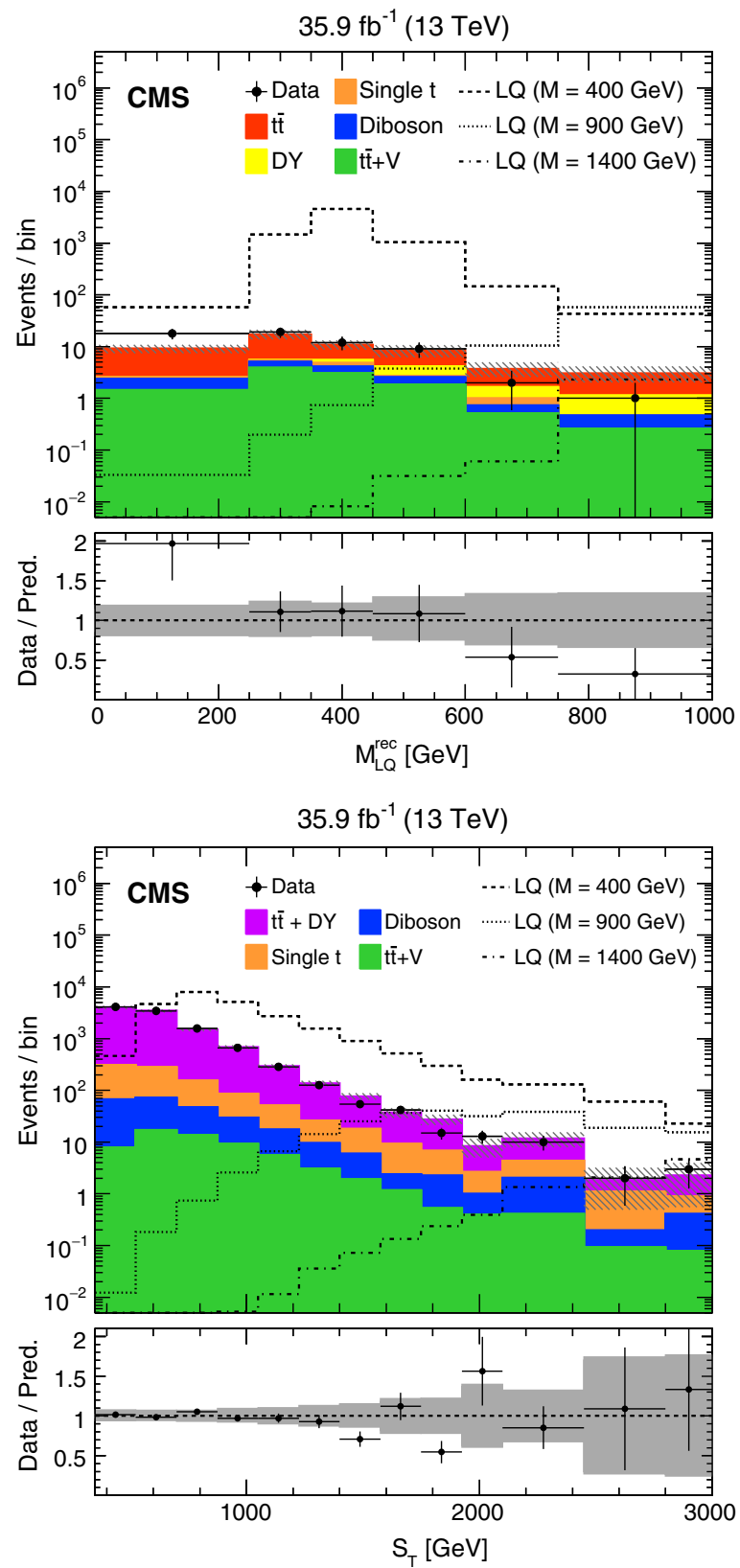

FIG. 1. Distributions for $M_{\mathrm{LQ}}^{\text {rec }}$ (category $A$, left) and $S_{T}$ (category $B$, right) after applying the full selection and estimating the $t \bar{t}$ and $\mathrm{DY}+$ jets background contributions from data in category $B$. All backgrounds are normalized according to the post-fit nuisance parameters based on the corresponding SM cross sections. In the upper panels, the hatched areas correspond to the total uncertainty. In the lower panels, the gray bands indicate the total uncertainty. parameter with a Gaussian distribution for each bin. A flat prior distribution is assumed for the signal cross section. The data are found to be compatible with the SM prediction in both categories. The distributions of $M_{\mathrm{LQ}}^{\mathrm{rec}}$ and $S_{T}$ after the background-only fit are shown in Fig. 1. A Bayesian method [93-95] is used to set upper limits at 95\% confidence level (C.L.) on the cross section for pair production of LQs decaying into a top quark and a muon. Pseudoexperiments are performed to determine the median along with the regions expected to contain $68 \%$ and $95 \%$ of the distribution of limits under the background-only hypothesis.
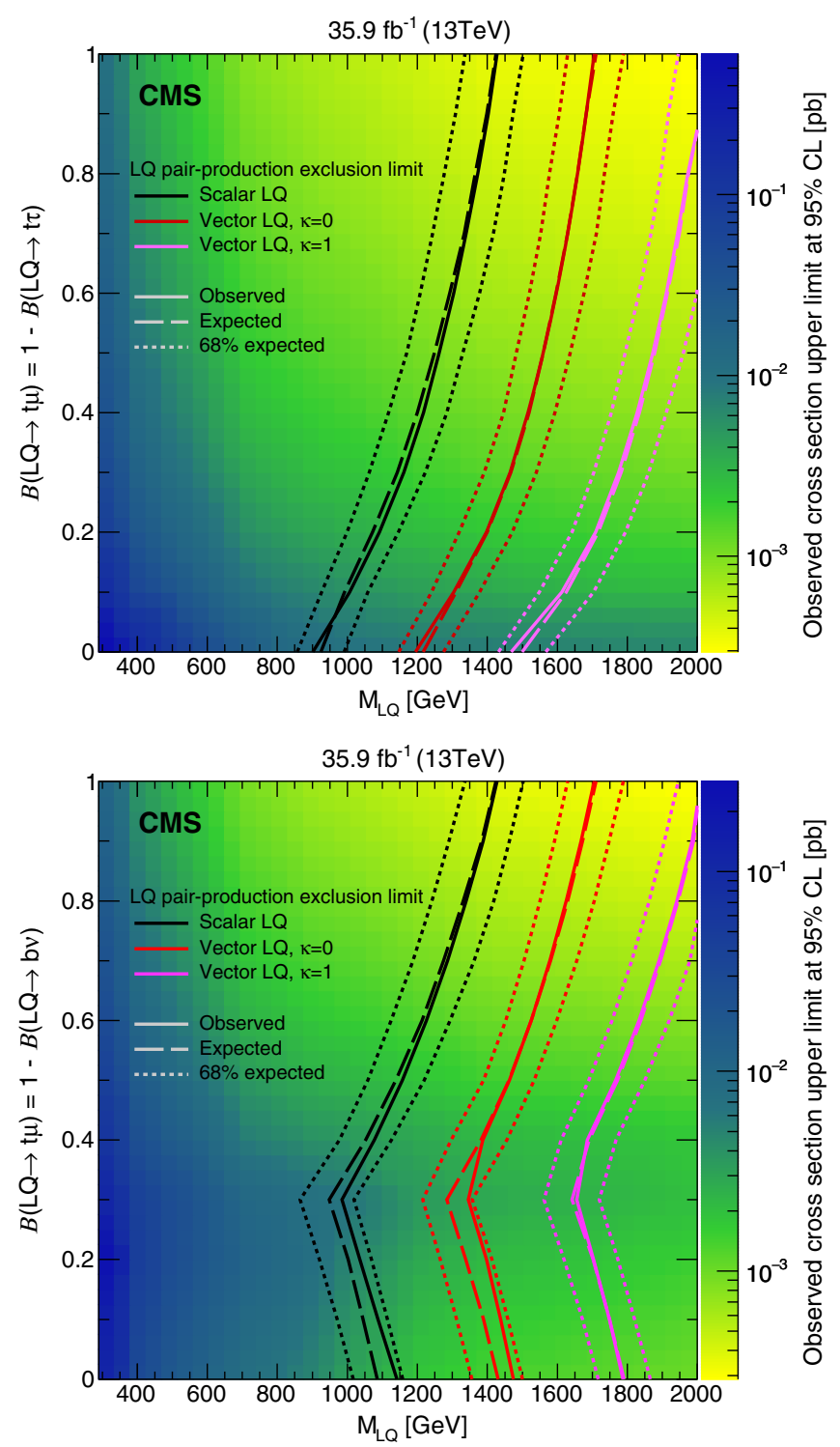

FIG. 2. Observed upper limits on the production cross section for pair production of LQs decaying into a top quark and a muon or a $\tau$ lepton (upper) and LQs decaying into a top quark and a muon or into a bottom quark and a neutrino (lower) at 95\% C.L. in the $M_{\mathrm{LQ}}-\mathcal{B}(\mathrm{LQ} \rightarrow t \mu)$ plane. The lines show the lower mass exclusion limits for scalar (black) and vector (colored) LQs. They are derived by using the prediction for the scalar and vector LQ signal calculated at NLO [50] and LO [51], respectively. 
Pair-produced scalar LQs decaying exclusively into a top quark and a muon, $\mathcal{B}(\mathrm{LQ} \rightarrow t \mu)=1$, are excluded at 95\% C.L. for LQ masses up to $1420 \mathrm{GeV}$, exceeding the best previous limit, obtained from a reinterpretation [36] of a search for supersymmetry [96], by more than $600 \mathrm{GeV}$. These results are combined with results from the LQ $\rightarrow t \tau$ [48] and LQ $\rightarrow b \nu$ [49] decay channels to set exclusions limits in the plane of $M_{\mathrm{LQ}}$ and $\mathcal{B}(\mathrm{LQ} \rightarrow t \mu)$. Figure 2 presents upper limits on the product of the production cross section and the branching fraction squared for $\mathcal{B}(\mathrm{LQ} \rightarrow t \mu)=1-\mathcal{B}(\mathrm{LQ} \rightarrow t \tau)$ (upper) and $\mathcal{B}(\mathrm{LQ} \rightarrow t \mu)=$ $1-\mathcal{B}(\mathrm{LQ} \rightarrow b \nu)$ (lower). The values for $\mathcal{B}(\mathrm{LQ} \rightarrow t \mu)=0$ correspond to the results of the search for pair-produced LQs in the LQ $\rightarrow t \tau$ decay channel (upper) and the search for pair-produced LQs in the LQ $\rightarrow b \nu$ channel (lower). These analyses excluded pair-produced scalar LQs in the targeted decay channels up to $M_{\mathrm{LQ}}=900$ and $1080 \mathrm{GeV}$, respectively. In the upper (lower) part of Fig. 2 the sensitivity is driven by the present analysis for values of $\mathcal{B}(\mathrm{LQ} \rightarrow t \mu)>0.1(0.3)$ and by the LQ $\rightarrow t \tau(b \nu)$ search for smaller values. Scalar LQs decaying into a top quark and either a muon or a $\tau$ lepton are excluded below masses of $900 \mathrm{GeV}$ for all values of $\mathcal{B}(\mathrm{LQ} \rightarrow t \mu)$, whereas LQs decaying either into a top quark and a muon or into a bottom quark and a neutrino are excluded up to $M_{\mathrm{LQ}}=$ $980 \mathrm{GeV}$. The simulated samples of scalar LQ pair production are also used to derive mass exclusion limits for pair-produced vector LQs, as the acceptance for both types of LQs is similar. The lower limit of excluded vector LQ masses is shown in Fig. 2 for the two coupling cases $\kappa=1$ and $\kappa=0$. Vector LQs are excluded up to masses of $1190 \mathrm{GeV}$ for all values of $\mathcal{B}(\mathrm{LQ} \rightarrow t \mu)$ and $\kappa$ considered.

In summary, this analysis represents the first search for leptoquarks decaying to top quarks and muons, reaching LQ masses of $\mathcal{O}(1 \mathrm{TeV})$ and placing direct constraints on the corresponding LQ coupling, thus probing the region of interest of models including LQs. With this result, all relevant couplings of LQs with an electric charge of $-1 / 3$ to third-generation quarks are examined for the first time.

We congratulate our colleagues in the CERN accelerator departments for the excellent performance of the LHC and thank the technical and administrative staffs at CERN and at other CMS institutes for their contributions to the success of the CMS effort. In addition, we gratefully acknowledge the computing centers and personnel of the Worldwide LHC Computing Grid for delivering so effectively the computing infrastructure essential to our analyses. Finally, we acknowledge the enduring support for the construction and operation of the LHC and the CMS detector provided by the following funding agencies: BMWFW and FWF (Austria); FNRS and FWO (Belgium); CNPq, CAPES, FAPERJ, and FAPESP (Brazil); MES (Bulgaria); CERN; CAS, MoST, and NSFC (China); COLCIENCIAS (Colombia); MSES and CSF (Croatia); RPF (Cyprus);
SENESCYT (Ecuador); MoER, ERC IUT, and ERDF (Estonia); Academy of Finland, MEC, and HIP (Finland); CEA and CNRS/IN2P3 (France); BMBF, DFG, and HGF (Germany); GSRT (Greece); OTKA and NIH (Hungary); DAE and DST (India); IPM (Iran); SFI (Ireland); INFN (Italy); MSIP and NRF (Republic of Korea); LAS (Lithuania); MOE and UM (Malaysia); BUAP, CINVESTAV, CONACYT, LNS, SEP, and UASLP-FAI (Mexico); MBIE (New Zealand); PAEC (Pakistan); MSHE and NSC (Poland); FCT (Portugal); JINR (Dubna); MON, RosAtom, RAS, RFBR and RAEP (Russia); MESTD (Serbia); SEIDI, CPAN, PCTI and FEDER (Spain); Swiss Funding Agencies (Switzerland); MST (Taipei); ThEPCenter, IPST, STAR, and NSTDA (Thailand); TUBITAK and TAEK (Turkey); NASU and SFFR (Ukraine); STFC (United Kingdom); DOE and NSF (USA).

[1] J. P. Lees et al. (BABAR Collaboration), Evidence for an Excess of $\bar{B} \rightarrow D^{(*)} \tau^{-} \bar{\nu}_{\tau}$ Decays, Phys. Rev. Lett. 109, 101802 (2012).

[2] J. P. Lees et al. (BABAR Collaboration), Measurement of an excess of $\bar{B} \rightarrow d^{(*)} \tau^{-} \bar{\nu}_{\tau}$ decays and implications for charged Higgs bosons, Phys. Rev. D 88, 072012 (2013).

[3] A. Matyja et al. (Belle Collaboration), Observation of $B^{0} \rightarrow D^{*-} \tau^{+} \nu_{\tau}$ Decay at Belle, Phys. Rev. Lett. 99, 191807 (2007).

[4] A. Bozek et al. (Belle Collaboration), Observation of $B^{+} \rightarrow \bar{D}^{* 0} \tau^{+} \nu_{\tau}$ and evidence for $B^{+} \rightarrow \bar{D}^{0} \tau^{+} \nu_{\tau}$ at Belle, Phys. Rev. D 82, 072005 (2010).

[5] M. Huschle et al. (Belle Collaboration), Measurement of the branching ratio of $\bar{B} \rightarrow D^{(*)} \tau^{-} \bar{\nu}_{\tau}$ relative to $\bar{B} \rightarrow D^{(*)} \ell^{-} \bar{\nu}_{\ell}$ decays with hadronic tagging at Belle, Phys. Rev. D 92, 072014 (2015).

[6] LHCb Collaboration, Measurement of the Ratio of Branching Fractions $\mathcal{B}\left(\bar{B}^{0} \rightarrow D^{*+} \tau^{-} \bar{\nu}_{\tau}\right) / \mathcal{B}\left(\bar{B}^{0} \rightarrow D^{*+} \mu^{-} \bar{\nu}_{\mu}\right)$, Phys. Rev. Lett. 115, 111803 (2015); Erratum, Phys. Rev. Lett. 115, 159901(E) (2015).

[7] LHCb Collaboration, Test of lepton flavor universality by the measurement of the $B^{0} \rightarrow D^{*-} \tau^{+} \nu_{\tau}$ branching fraction using three-prong $\tau$ decays, Phys. Rev. D 97, 072013 (2018).

[8] Y. Amhis et al. (HFLAV Collaboration), Averages of $b$-hadron, $c$-hadron, and $\tau$-lepton properties as of summer 2016, Eur. Phys. J. C 77, 895 (2017).

[9] LHCb Collaboration, Test of Lepton Universality Using $B^{+} \rightarrow K^{+} \ell^{+} \ell^{-}$Decays, Phys. Rev. Lett. 113, 151601 (2014).

[10] LHCb Collaboration, Differential branching fractions and isospin asymmetries of $B \rightarrow K^{(*)} \mu^{+} \mu^{-}$decays, J. High Energy Phys. 06 (2014) 133.

[11] LHCb Collaboration, Angular analysis of the $B^{0} \rightarrow K^{* 0} \mu^{+} \mu^{-}$ decay using $3 \mathrm{fb}^{-1}$ of integrated luminosity, J. High Energy Phys. 02 (2016) 104.

[12] LHCb Collaboration, Test of lepton universality with $B^{0} \rightarrow K^{* 0} \ell^{+} \ell^{-}$decays, J. High Energy Phys. 08 (2017) 055. 
[13] G. W. Bennett et al. (Muon g-2), Final report of the muon E821 anomalous magnetic moment measurement at BNL, Phys. Rev. D 73, 072003 (2006).

[14] M. Davier, A. Hoecker, B. Malaescu, and Z. Zhang, Reevaluation of the hadronic vacuum polarisation contributions to the standard model predictions of the muon $g-2$ and $\alpha\left(m_{Z}^{2}\right)$ using newest hadronic cross-section data, Eur. Phys. J. C 77, 827 (2017).

[15] M. Tanaka and R. Watanabe, New physics in the weak interaction of $\bar{B} \rightarrow D^{(*)} \tau \bar{\nu}$, Phys. Rev. D 87, 034028 (2013).

[16] Y. Sakaki, M. Tanaka, A. Tayduganov, and R. Watanabe, Testing leptoquark models in $\bar{B} \rightarrow D^{(*)} \tau \bar{\nu}$, Phys. Rev. D 88, 094012 (2013).

[17] I. Doršner, S. Fajfer, N. Košnik, and I. Nišandžić, Minimally flavored colored scalar in $\bar{B} \rightarrow D^{(*)} \tau \bar{\nu}$ and the mass matrices constraints, J. High Energy Phys. 11 (2013) 084.

[18] B. Dumont, K. Nishiwaki, and R. Watanabe, LHC constraints and prospects for $S_{1}$ scalar leptoquark explaining the $\bar{B} \rightarrow D^{(*)} \tau \bar{\nu}$ anomaly, Phys. Rev. D 94, 034001 (2016).

[19] A. Crivellin, D. Müller, and T. Ota, Simultaneous explanation of $R\left(D^{(*)}\right)$ and $b \rightarrow \mathrm{s} \mu^{+} \mu^{-}$: the last scalar leptoquarks standing, J. High Energy Phys. 09 (2017) 040.

[20] B. Gripaios, M. Nardecchia, and S. A. Renner, Composite leptoquarks and anomalies in $b$-meson decays, J. High Energy Phys. 05 (2015) 006.

[21] M. Bauer and M. Neubert, Minimal Leptoquark Explanation for the $R_{D^{(*)}}, R_{K}$, and $(g-2)_{\mu}$ Anomalies, Phys. Rev. Lett. 116, 141802 (2016).

[22] E. C. Leskow, G. D'Ambrosio, A. Crivellin, and D. Müller, $(g-2)_{\mu}$, lepton flavor violation, and Z decays with leptoquarks: Correlations and future prospects, Phys. Rev. D 95, 055018 (2017).

[23] D. Bečirević and O. Sumensari, A leptoquark model to accommodate $R_{K}^{\exp }<R_{K}^{\mathrm{SM}}$ and $R_{K^{*}}^{\exp }<R_{K^{*}}^{\mathrm{SM}}$, J. High Energy Phys. 08 (2017) 104.

[24] G. Hiller and I. Nišandžić, $R_{K}$ and $R_{K^{*}}$ beyond the standard model, Phys. Rev. D 96, 035003 (2017).

[25] G. Hiller, D. Loose, and I. Nišandžić, Flavorful leptoquarks at hadron colliders, Phys. Rev. D 97, 075004 (2018).

[26] J. C. Pati and A. Salam, Lepton number as the fourth color, Phys. Rev. D 10, 275 (1974); Erratum, Phys. Rev. D 11, 703 (E) (1975).

[27] H. Georgi and S. L. Glashow, Unity of All ElementaryParticle Forces, Phys. Rev. Lett. 32, 438 (1974).

[28] H. Fritzsch and P. Minkowski, Unified interactions of leptons and hadrons, Ann. Phys. (N.Y.) 93, 193 (1975).

[29] E. Farhi and L. Susskind, Technicolor, Phys. Rep. 74, 277 (1981).

[30] K. Lane and M. V. Ramana, Walking technicolor signatures at hadron colliders, Phys. Rev. D 44, 2678 (1991).

[31] B. Schrempp and F. Schrempp, Light leptoquarks, Phys. Lett. 153B, 101 (1985).

[32] B. Gripaios, Composite leptoquarks at the LHC, J. High Energy Phys. 02 (2010) 045.

[33] W. Buchmüller, R. Rückl, and D. Wyler, Leptoquarks in lepton-quark collisions, Phys. Lett. B 191, 442 (1987); Erratum, Phys. Lett. B 448, 320(E) (1999).

[34] D. E. Acosta and S. K. Blessing, Leptoquark searches at HERA and the tevatron, Annu. Rev. Nucl. Part. Sci. 49, 389 (1999).
[35] I. Doršner, S. Fajfer, A. Greljo, J. F. Kamenik, and N. Košnik, Physics of leptoquarks in precision experiments and at particle colliders, Phys. Rep. 641, 1 (2016).

[36] B. Diaz, M. Schmaltz, and Y.-M. Zhong, The leptoquark hunter's guide: Pair production, J. High Energy Phys. 10 (2017) 097.

[37] CMS Collaboration, Search for third-generation scalar leptoquarks in the $t \tau$ channel in proton-proton collisions at $\sqrt{s}=8 \mathrm{TeV}$, J. High Energy Phys. 07 (2015) 042; Erratum, J. High Energy Phys. 11 (2016) 056.

[38] V. M. Abazov et al. (D0 Collaboration), Search for ThirdGeneration Leptoquarks in $\mathrm{p} \overline{\mathrm{p}}$ Collisions at $\sqrt{s}=1.96 \mathrm{TeV}$, Phys. Rev. Lett. 99, 061801 (2007).

[39] T. Aaltonen et al. (CDF), Search for third generation vector leptoquarks in $\mathrm{p} \overline{\mathrm{p}}$ collisions at $\sqrt{s}=1.96 \mathrm{TeV}$, Phys. Rev. D 77, 091105 (2008).

[40] ATLAS Collaboration, Search for third generation scalar leptoquarks in $\mathrm{pp}$ collisions at $\sqrt{s}=7 \mathrm{TeV}$ with the ATLAS detector, J. High Energy Phys. 06 (2013) 033.

[41] CMS Collaboration, Search for third-generation leptoquarks and scalar bottom quarks in pp collisions at $\sqrt{s}=7 \mathrm{TeV}$, J. High Energy Phys. 12 (2012) 055.

[42] CMS Collaboration, Search for pair production of thirdgeneration scalar leptoquarks and top squarks in protonproton collisions at $\sqrt{s}=8 \mathrm{TeV}$, Phys. Lett. B 739, 229 (2014).

[43] CMS Collaboration, Searches for third-generation squark production in fully hadronic final states in proton-proton collisions at $\sqrt{s}=8 \mathrm{TeV}$, J. High Energy Phys. 06 (2015) 116.

[44] ATLAS Collaboration, Searches for scalar leptoquarks in pp collisions at $\sqrt{s}=8 \mathrm{TeV}$ with the ATLAS detector, Eur. Phys. J. C 76, 5 (2016).

[45] CMS Collaboration, Search for heavy neutrinos or thirdgeneration leptoquarks in final states with two hadronically decaying $\tau$ leptons and two jets in proton-proton collisions at $\sqrt{s}=13 \mathrm{TeV}$, J. High Energy Phys. 03 (2017) 077.

[46] CMS Collaboration, Search for third-generation scalar leptoquarks and heavy right-handed neutrinos in final states with two tau leptons and two jets in proton-proton collisions at $\sqrt{s}=13 \mathrm{TeV}$, J. High Energy Phys. 07 (2017) 121.

[47] CMS Collaboration, Search for new phenomena with the $M_{T 2}$ variable in the all-hadronic final state produced in proton-proton collisions at $\sqrt{s}=13 \mathrm{TeV}$, Eur. Phys. J. C 77, 710 (2017).

[48] CMS Collaboration, Search for third-generation scalar leptoquarks decaying to a top quark and a $\tau$ lepton at $\sqrt{s}=13 \mathrm{TeV}$, Eur. Phys. J. C 78, 707 (2018).

[49] CMS Collaboration, Constraints on models of scalar and vector leptoquarks decaying to a quark and a neutrino at $\sqrt{s}=13$ TeV, Phys. Rev. D 98, 032005 (2018).

[50] M. Kramer, T. Plehn, M. Spira, and P. M. Zerwas, Pair production of scalar leptoquarks at the CERN LHC, Phys. Rev. D 71, 057503 (2005).

[51] I. Doršner and A. Greljo, Leptoquark toolbox for precision collider studies, J. High Energy Phys. 05 (2018) 126.

[52] CMS Collaboration, The CMS experiment at the CERN LHC, J. Instrum. 3, S08004 (2008).

[53] CMS Collaboration, The CMS trigger system, J. Instrum. 12, P01020 (2017). 
[54] T. Sjöstrand, S. Mrenna, and P.Z. Skands, PYTHIA 6.4 physics and manual, J. High Energy Phys. 05 (2006) 026.

[55] T. Sjöstrand, S. Ask, J. R. Christiansen, R. Corke, N. Desai, P. Ilten, S. Mrenna, S. Prestel, C. O. Rasmussen, and P.Z. Skands, An introduction to PYTHIA 8.2, Comput. Phys. Commun. 191, 159 (2015).

[56] P. Nason, A new method for combining NLO QCD with shower Monte Carlo algorithms, J. High Energy Phys. 11 (2004) 040.

[57] S. Frixione, P. Nason, and Carlo Oleari, Matching NLO QCD computations with parton shower simulations: The POWHEG method, J. High Energy Phys. 11 (2007) 070.

[58] S. Alioli, P. Nason, C. Oleari, and E. Re, A general framework for implementing NLO calculations in shower Monte Carlo programs: The POWHEG BOX, J. High Energy Phys. 06 (2010) 043.

[59] S. Frixione, P. Nason, and G. Ridolfi, A positive-weight next-to-leading-order Monte Carlo for heavy flavour hadroproduction, J. High Energy Phys. 09 (2007) 126.

[60] T. Melia, P. Nason, R. Rontsch, and G. Zanderighi, $\mathrm{W}^{+} \mathrm{W}^{-}$, WZ and ZZ production in the POWHEG BOX, J. High Energy Phys. 11 (2011) 078.

[61] P. Nason and G. Zanderighi, $\mathrm{W}^{+} \mathrm{W}^{-}$, $\mathrm{WZ}$ and $\mathrm{ZZ}$ production in the POWHEG-BOX-V2, Eur. Phys. J. C 74, 2702 (2014).

[62] S. Alioli, P. Nason, C. Oleari, and E. Re, NLO single-top production matched with shower in POWHEG: $s$ - and $t$-channel contributions, J. High Energy Phys. 09 (2009) 111; Erratum, J. High Energy Phys. 02 (2010) 11.

[63] E. Re, Single-top Wt-channel production matched with parton showers using the POWHEG method, Eur. Phys. J. C 71, 1547 (2011).

[64] J. Alwall, R. Frederix, S. Frixione, V. Hirschi, F. Maltoni, O. Mattelaer, H. S. Shao, T. Stelzer, P. Torrielli, and M. Zaro, The automated computation of tree-level and next-to-leading order differential cross sections, and their matching to parton shower simulations, J. High Energy Phys. 07 (2014) 079.

[65] R. Frederix and S. Frixione, Merging meets matching in MC@NLO, J. High Energy Phys. 12 (2012) 061.

[66] J. Alwall, S. Hoeche, F. Krauss, N. Lavesson, L. Lonnblad, F. Maltoni, M. L. Mangano, M. Moretti, C. G. Papadopoulos, F. Piccinini, S. Schumann, M. Treccani, J. Winter, and M. Worek, Comparative study of various algorithms for the merging of parton showers and matrix elements in hadronic collisions, Eur. Phys. J. C 53, 473 (2008).

[67] R. D. Ball et al. (NNPDF), Parton distributions for the LHC Run II, J. High Energy Phys. 04 (2015) 040.

[68] CMS Collaboration, Investigations of the impact of the parton shower tuning in PYTHIA 8 in the modelling of $t \bar{t}$ at $\sqrt{s}=8$ and $13 \mathrm{TeV}$, CMS Physics Analysis Summary Report No. CMS-PAS-TOP-16-021, 2016, https://cds.cern .ch/record/2235192.

[69] CMS Collaboration, Event generator tunes obtained from underlying event and multiparton scattering measurements, Eur. Phys. J. C 76, 155 (2016).

[70] P. Skands, S. Carrazza, and J. Rojo, Tuning PYTHIA 8.1: the Monash 2013 Tune, Eur. Phys. J. C 74, 3024 (2014).

[71] S. Agostinelli et al. (GEANT4 Collaboration), GEANT4A simulation toolkit, Nucl. Instrum. Methods Phys. Res., Sect. A 506, 250 (2003).
[72] J. Allison et al., GEANT4 developments and applications, IEEE Trans. Nucl. Sci. 53, 270 (2006).

[73] CMS Collaboration, Particle-flow reconstruction and global event description with the CMS detector, J. Instrum. 12, P10003 (2017).

[74] M. Cacciari, G. P. Salam, and G. Soyez, The anti- $k_{T}$ jet clustering algorithm, J. High Energy Phys. 04 (2008) 063.

[75] M. Cacciari, G. P. Salam, and G. Soyez, FastJet user manual, Eur. Phys. J. C 72, 1896 (2012).

[76] CMS Collaboration (CMS Collaboration), Technical Proposal for the Phase-II upgrade of the Compact Muon Solenoid, CMS Technical proposal Report Nos. CERNLHCC-2015-010, CMS-TDR-15-02, 2015, http://cds.cern .ch/record/2020886.

[77] CMS Collaboration, Performance of the CMS muon detector and muon reconstruction with proton-proton collisions at $\sqrt{s}=13 \mathrm{TeV}$, J. Instrum. 13, P06015 (2018).

[78] CMS Collaboration, Performance of photon reconstruction and identification with the CMS detector in protonproton collisions at $\sqrt{s}=8 \mathrm{TeV}$, J. Instrum. 10, P08010 (2015).

[79] CMS Collaboration, Jet energy scale and resolution in the CMS experiment in pp collisions at $8 \mathrm{TeV}$, J. Instrum. 12, P02014 (2017).

[80] CMS Collaboration, Identification of heavy-flavour jets with the CMS detector in pp collisions at $13 \mathrm{TeV}$, J. Instrum. 13, P05011 (2018).

[81] J. Butterworth et al., PDF4LHC recommendations for LHC Run II, J. Phys. G 43, 023001 (2016).

[82] CMS Collaboration, Measurement of the $t \bar{t}$ production cross section using events in the $e \mu$ final state in pp collisions at $\sqrt{s}=13$ TeV, Eur. Phys. J. C 77, 172 (2017).

[83] CMS Collaboration, Measurement of Inclusive $W$ and $Z$ Boson Production Cross Sections in pp Collisions at $\sqrt{s}=8 \mathrm{TeV}$, Phys. Rev. Lett. 112, 191802 (2014).

[84] CMS Collaboration, Cross section measurement of $t$-channel single top quark production in pp collisions at $\sqrt{s}=13 \mathrm{TeV}$, Phys. Lett. B 772, 752 (2017).

[85] N. Kidonakis, NNLL threshold resummation for top-pair and single-top production, Phys. Part. Nucl. 45, 714 (2014).

[86] CMS Collaboration, Observation of the Associated Production of a Single Top Quark and a $W$ Boson in $p p$ Collisions at $\sqrt{s}=8 \mathrm{TeV}$, Phys. Rev. Lett. 112, 231802 (2014).

[87] T. Gehrmann, M. Grazzini, S. Kallweit, P. Maierhöfer, A. von Manteuffel, S. Pozzorini, D. Rathlev, and L. Tancredi, $W^{+} W^{-}$Production at Hadron Colliders in Next to Next to Leading Order QCD, Phys. Rev. Lett. 113, 212001 (2014).

[88] J. M. Campbell, R. K. Ellis, and C. Williams, Vector boson pair production at the LHC, J. High Energy Phys. 07 (2011) 018.

[89] CMS Collaboration, Measurement of the WZ production cross section in pp collisions at $\sqrt{s}=13 \mathrm{TeV}$, Phys. Lett. B 766, 268 (2017).

[90] CMS Collaboration, Measurement of the cross section for top quark pair production in association with a $\mathrm{W}$ or $\mathrm{Z}$ boson in proton-proton collisions at $\sqrt{s}=13 \mathrm{TeV}, \mathrm{J}$. High Energy Phys. 08 (2018) 011.

[91] CMS Collaboration (CMS Collaboration), CMS Luminosity Measurements for the 2016 Data Taking Period, 
CMS Physics Analysis Summary CMS-PAS-LUM-17-001, 2017, http://cds.cern.ch/record/2257069.

[92] CMS Collaboration, Measurement of the inelastic protonproton cross section at $\sqrt{s}=13 \mathrm{TeV}$, J. High Energy Phys. 07 (2018) 161.

[93] J. Ott, THETA-A framework for template-based modeling and inference, 2010, http://www-ekp.physik.uni-karlsruhe .de/ ott/theta/theta-auto/.
[94] A. O'Hagan and J. J Forster, Kendall's Advanced Theory of Statistics. Vol. 2B: Bayesian Inference (Arnold, London, 2004).

[95] G. Cowan, Statistics, Chin. Phys. C 40, 100001 (2016).

[96] CMS Collaboration, Search for supersymmetry in events with at least three electrons or muons, jets, and missing transverse momentum in proton-proton collisions at $\sqrt{s}=13 \mathrm{TeV}$, J. High Energy Phys. 02 (2018) 067.

A. M. Sirunyan, ${ }^{1}$ A. Tumasyan, ${ }^{1}$ W. Adam, ${ }^{2}$ F. Ambrogi, ${ }^{2}$ E. Asilar, ${ }^{2}$ T. Bergauer, ${ }^{2}$ J. Brandstetter, ${ }^{2}$ M. Dragicevic, ${ }^{2}$ J. Erö, ${ }^{2}$ A. Escalante Del Valle, ${ }^{2}$ M. Flechl, ${ }^{2}$ R. Frühwirth, ${ }^{2, b}$ V. M. Ghete, ${ }^{2}$ J. Hrubec, ${ }^{2}$ M. Jeitler, ${ }^{2, b}$ N. Krammer, ${ }^{2}$ I. Krätschmer, ${ }^{2}$ D. Liko, ${ }^{2}$ T. Madlener, ${ }^{2}$ I. Mikulec, ${ }^{2}$ N. Rad, ${ }^{2}$ H. Rohringer, ${ }^{2}$ J. Schieck ${ }^{2, b}$ R. Schöfbeck, ${ }^{2}$ M. Spanring, ${ }^{2}$ D. Spitzbart, ${ }^{2}$ A. Taurok, ${ }^{2}$ W. Waltenberger, ${ }^{2}$ J. Wittmann, ${ }^{2}$ C.-E. Wulz, ${ }^{2, b}$ M. Zarucki, ${ }^{2}$ V. Chekhovsky, ${ }^{3}$ V. Mossolov, ${ }^{3}$ J. Suarez Gonzalez, ${ }^{3}$ E. A. De Wolf, ${ }^{4}$ D. Di Croce, ${ }^{4}$ X. Janssen, ${ }^{4}$ J. Lauwers, ${ }^{4}$ M. Pieters ${ }^{4}$ H. Van Haevermaet, ${ }^{4}$ P. Van Mechelen, ${ }^{4}$ N. Van Remortel, ${ }^{4}$ S. Abu Zeid, ${ }^{5}$ F. Blekman, ${ }^{5}$ J. D'Hondt, ${ }^{5}$ I. De Bruyn, ${ }^{5}$ J. De Clercq, ${ }^{5}$ K. Deroover, ${ }^{5}$ G. Flouris, ${ }^{5}$ D. Lontkovskyi, ${ }^{5}$ S. Lowette, ${ }^{5}$ I. Marchesini, ${ }^{5}$ S. Moortgat, ${ }^{5}$ L. Moreels, ${ }^{5}$ Q. Python, ${ }^{5}$ K. Skovpen, ${ }^{5}$ S. Tavernier, ${ }^{5}$ W. Van Doninck, ${ }^{5}$ P. Van Mulders, ${ }^{5}$ I. Van Parijs, ${ }^{5}$ D. Beghin, ${ }^{6}$ B. Bilin, ${ }^{6}$ H. Brun, ${ }^{6}$ B. Clerbaux,${ }^{6}$ G. De Lentdecker, ${ }^{6}$ H. Delannoy, ${ }_{6}^{6}$ B. Dorney, ${ }^{6}$ G. Fasanella, ${ }^{6}$ L. Favart, ${ }^{6}$ R. Goldouzian, ${ }^{6}$ A. Grebenyuk, ${ }^{6}$ A. K. Kalsi, ${ }^{6}$ T. Lenzi, ${ }^{6}$ J. Luetic, ${ }^{6}$ N. Postiau, ${ }^{6}$ E. Starling, ${ }^{6}$ L. Thomas, ${ }^{6}$ C. Vander Velde, ${ }^{6}$ P. Vanlaer, ${ }^{6}$ D. Vannerom, ${ }^{6}$ Q. Wang, ${ }^{6}$ T. Cornelis, ${ }^{7}$ D. Dobur, ${ }^{7}$ A. Fagot, ${ }^{7}$ M. Gul, ${ }^{7}$ I. Khvastunov, ${ }^{7, c}$ D. Poyraz, ${ }^{7}$ C. Roskas, ${ }^{7}$ D. Trocino, ${ }^{7}$ M. Tytgat, ${ }^{7}$ W. Verbeke, ${ }^{7}$ B. Vermassen, ${ }^{7}$ M. Vit, ${ }^{7}$ N. Zaganidis, ${ }^{7}$ H. Bakhshiansohi, ${ }^{8}$ O. Bondu, ${ }^{8}$ S. Brochet, ${ }^{8}$ G. Bruno, ${ }^{8}$ C. Caputo, ${ }^{8}$ P. David, ${ }^{8}$ C. Delaere, ${ }^{8}$ M. Delcourt, ${ }^{8}$ A. Giammanco, ${ }^{8}$ G. Krintiras, ${ }^{8}$ V. Lemaitre, ${ }^{8}$ A. Magitteri, ${ }^{8}$ A. Mertens, ${ }^{8}$ M. Musich, ${ }^{8}$ K. Piotrzkowski, ${ }^{8}$ A. Saggio, ${ }^{8}$ M. Vidal Marono, ${ }^{8}$ S. Wertz, ${ }^{8}$ J. Zobec, ${ }^{8}$ F. L. Alves, ${ }^{9}$ G. A. Alves, ${ }^{9}$

M. Correa Martins Junior, ${ }^{9}$ G. Correia Silva, ${ }^{9}$ C. Hensel, ${ }^{9}$ A. Moraes, ${ }^{9}$ M. E. Pol, ${ }^{9}$ P. Rebello Teles, ${ }^{9}$

E. Belchior Batista Das Chagas, ${ }^{10}$ W. Carvalho, ${ }^{10}$ J. Chinellato, ${ }^{10, \mathrm{~d}}$ E. Coelho, ${ }^{10}$ E. M. Da Costa, ${ }^{10}$ G. G. Da Silveira, ${ }^{10, e}$ D. De Jesus Damiao, ${ }^{10}$ C. De Oliveira Martins, ${ }^{10}$ S. Fonseca De Souza,${ }^{10}$ H. Malbouisson, ${ }^{10}$ D. Matos Figueiredo, ${ }^{10}$ M. Melo De Almeida, ${ }^{10}$ C. Mora Herrera,${ }^{10}$ L. Mundim, ${ }^{10}$ H. Nogima, ${ }^{10}$ W. L. Prado Da Silva, ${ }^{10}$ L. J. Sanchez Rosas, ${ }^{10}$ A. Santoro, ${ }^{10}$ A. Sznajder, ${ }^{10}$ M. Thiel, ${ }^{10}$ E. J. Tonelli Manganote, ${ }^{10, d}$ F. Torres Da Silva De Araujo, ${ }^{10}$ A. Vilela Pereira, ${ }^{10}$ S. Ahuja ${ }^{11 \mathrm{a}}$ C. A. Bernardes, ${ }^{11 \mathrm{a}}$ L. Calligaris, ${ }^{11 \mathrm{a}}$ T. R. Fernandez Perez Tomei, ${ }^{11 \mathrm{a}}$ E. M. Gregores, ${ }^{11 \mathrm{a}, 11 \mathrm{~b}}$

P. G. Mercadante, ${ }^{11 \mathrm{a}, 11 \mathrm{~b}}$ S. F. Novaes, ${ }^{11 \mathrm{a}}$ Sandra S. Padula, ${ }^{11 \mathrm{a}}$ A. Aleksandrov, ${ }^{12}$ R. Hadjiiska, ${ }^{12}$ P. Iaydjiev, ${ }^{12}$ A. Marinov, ${ }^{12}$ M. Misheva, ${ }^{12}$ M. Rodozov, ${ }^{12}$ M. Shopova, ${ }^{12}$ G. Sultanov, ${ }^{12}$ A. Dimitrov, ${ }^{13}$ L. Litov, ${ }^{13}$ B. Pavlov,${ }^{13}$ P. Petkov,${ }^{13}$ W. Fang, ${ }^{14, f}$ X. Gao, ${ }^{14, \mathrm{f}}$ L. Yuan, ${ }^{14}$ M. Ahmad, ${ }^{15}$ J. G. Bian, ${ }^{15}$ G. M. Chen, ${ }^{15}$ H. S. Chen, ${ }^{15}$ M. Chen, ${ }^{15}$ Y. Chen, ${ }^{15}$ C. H. Jiang, ${ }^{15}$ D. Leggat ${ }_{15}^{15}$ H. Liao, ${ }^{15}$ Z. Liu, ${ }^{15}$ F. Romeo ${ }^{15}$ S. M. Shaheen, ${ }^{15, g}$ A. Spiezia, ${ }^{15}$ J. Tao, ${ }^{15}$ Z. Wang, ${ }^{15}$ E. Yazgan, ${ }^{15}$ H. Zhang ${ }^{15}$ S. Zhang, ${ }^{15, \mathrm{~g}}$ J. Zhao ${ }^{15}$ Y. Ban, ${ }^{16}$ G. Chen, ${ }^{16}$ A. Levin, ${ }^{16} \mathrm{~J} . \mathrm{Li},{ }^{16} \mathrm{~L} . \mathrm{Li},{ }^{16} \mathrm{Q} . \mathrm{Li},{ }^{16}$ Y. Mao, ${ }^{16} \mathrm{~S}$. J. Qian, ${ }^{16}$ D. Wang, ${ }^{16} \mathrm{Z} . \mathrm{Xu},{ }^{16}$ Y. Wang, ${ }^{17}$ C. Avila ${ }^{18}$ A. Cabrera, ${ }^{18}$ C. A. Carrillo Montoya,${ }^{18}$ L. F. Chaparro Sierra, ${ }^{18}$ C. Florez, ${ }^{18}$

C. F. González Hernández, ${ }^{18}$ M. A. Segura Delgado, ${ }^{18}$ B. Courbon, ${ }^{19}$ N. Godinovic, ${ }^{19}$ D. Lelas, ${ }^{19}$ I. Puljak,${ }^{19}$ T. Sculac, ${ }^{19}$ Z. Antunovic ${ }^{20}$ M. Kovac, ${ }^{20}$ V. Brigljevic, ${ }^{21}$ D. Ferencek, ${ }^{21}$ K. Kadija,${ }^{21}$ B. Mesic,${ }^{21}$ A. Starodumov, ${ }^{21, h}$ T. Susa, ${ }^{21}$ M. W. Ather, ${ }^{22}$ A. Attikis, ${ }^{22}$ M. Kolosova, ${ }^{22}$ G. Mavromanolakis, ${ }^{22}$ J. Mousa, ${ }^{22}$ C. Nicolaou, ${ }^{22}$ F. Ptochos,${ }^{22}$ P. A. Razis, ${ }^{22}$ H. Rykaczewski, ${ }^{22}$ M. Finger, ${ }^{23, i}$ M. Finger Jr., ${ }^{23, i}$ E. Ayala, ${ }^{24}$ E. Carrera Jarrin, ${ }^{25}$ A. Mahrous, ${ }^{26, j}$ A. Mohamed, ${ }^{26, k}$ E. Salama, ${ }^{26,1, \mathrm{~m}}$ S. Bhowmik, ${ }^{27}$ A. Carvalho Antunes De Oliveira, ${ }^{27}$ R. K. Dewanjee, ${ }^{27}$ K. Ehataht, ${ }^{27}$ M. Kadastik, ${ }^{27}$ M. Raidal, ${ }^{27}$ C. Veelken, ${ }^{27}$ P. Eerola,${ }^{28}$ H. Kirschenmann, ${ }^{28}$ J. Pekkanen, ${ }^{28}$ M. Voutilainen, ${ }^{28}$ J. Havukainen, ${ }^{29}$ J. K. Heikkilä, ${ }^{29}$ T. Järvinen, ${ }^{29}$ V. Karimäki, ${ }^{29}$ R. Kinnunen, ${ }^{29}$ T. Lampén, ${ }^{29}$ K. Lassila-Perini, ${ }^{29}$ S. Laurila, ${ }^{29}$ S. Lehti, ${ }^{29}$ T. Lindén, ${ }^{29}$ P. Luukka, ${ }^{29}$ T. Mäenpää, ${ }^{29}$ H. Siikonen, ${ }^{29}$ E. Tuominen, ${ }^{29}$ J. Tuominiemi, ${ }^{29}$ T. Tuuva, ${ }^{30}$ M. Besancon, ${ }^{31}$ F. Couderc, ${ }^{31}$ M. Dejardin, ${ }^{31}$ D. Denegri, ${ }^{31}$ J. L. Faure, ${ }^{31}$ F. Ferri, ${ }^{31}$ S. Ganjour, ${ }^{31}$ A. Givernaud, ${ }^{31}$ P. Gras, ${ }^{31}$ G. Hamel de Monchenault, ${ }^{31}$ P. Jarry, ${ }^{31}$ C. Leloup ${ }^{31}$ E. Locci ${ }^{31}$ J. Malcles,${ }^{31}$ G. Negro, ${ }^{31}$ J. Rander, ${ }^{31}$ A. Rosowsky, ${ }^{31}$ M. Ö. Sahin, ${ }^{31}$ M. Titov, ${ }^{31}$ A. Abdulsalam, ${ }^{32, n}$ C. Amendola,${ }^{32}$ I. Antropov, ${ }^{32}$ F. Beaudette, ${ }^{32}$ P. Busson, ${ }^{32}$ C. Charlot, ${ }^{32}$ R. Granier de Cassagnac, ${ }^{32}$ I. Kucher, ${ }^{32}$ A. Lobanov, ${ }^{32}$ J. Martin Blanco, ${ }^{32}$ C. Martin Perez, ${ }^{32}$ M. Nguyen, ${ }^{32}$ C. Ochando ${ }^{32}$ G. Ortona, ${ }^{32}$ P. Paganini, ${ }^{32}$ P. Pigard, ${ }^{32}$ J. Rembser, ${ }^{32}$ R. Salerno, ${ }^{32}$ J. B. Sauvan, ${ }^{32}$ Y. Sirois, ${ }^{32}$ A. G. Stahl Leiton, ${ }^{32}$ 
A. Zabi, ${ }^{32}$ A. Zghiche, ${ }^{32}$ J.-L. Agram, ${ }^{33,0}$ J. Andrea, ${ }^{33}$ D. Bloch, ${ }^{33}$ J.-M. Brom,${ }^{33}$ E. C. Chabert, ${ }^{33}$ V. Cherepanov, ${ }^{33}$ C. Collard, ${ }^{33}$ E. Conte, ${ }^{33,0}$ J.-C. Fontaine ${ }^{33,0}$ D. Gelé, ${ }^{33}$ U. Goerlach, ${ }^{33}$ M. Jansová, ${ }^{33}$ A.-C. Le Bihan, ${ }^{33}$ N. Tonon, ${ }^{33}$ P. Van Hove, ${ }^{33}$ S. Gadrat, ${ }^{34}$ S. Beauceron, ${ }^{35}$ C. Bernet, ${ }^{35}$ G. Boudoul, ${ }^{35}$ N. Chanon, ${ }^{35}$ R. Chierici, ${ }^{35}$ D. Contardo, ${ }^{35}$ P. Depasse, ${ }^{35}$ H. El Mamouni, ${ }^{35}$ J. Fay, ${ }^{35}$ L. Finco, ${ }^{35}$ S. Gascon, ${ }^{35}$ M. Gouzevitch, ${ }^{35}$ G. Grenier, ${ }^{35}$ B. Ille,${ }^{35}$ F. Lagarde ${ }^{35}$ I. B. Laktineh, ${ }^{35}$ H. Lattaud, ${ }^{35}$ M. Lethuillier, ${ }^{35}$ L. Mirabito, ${ }^{35}$ S. Perries, ${ }^{35}$ A. Popov, ${ }^{35, p}$ V. Sordini, ${ }^{35}$ G. Touquet, ${ }^{35}$ M. Vander Donckt, ${ }^{35}$ S. Viret, ${ }^{35}$ T. Toriashvilii, ${ }^{36, q}$ Z. Tsamalaidze, ${ }^{37, \mathrm{i}}$ C. Autermann, ${ }^{38}$ L. Feld,${ }^{38}$ M. K. Kiesel, ${ }^{38}$ K. Klein,${ }^{38}$ M. Lipinski, ${ }^{38}$ M. Preuten, ${ }^{38}$ M. P. Rauch, ${ }^{38}$ C. Schomakers, ${ }^{38}$ J. Schulz, ${ }^{38}$ M. Teroerde, ${ }^{38}$ B. Wittmer, ${ }^{38}$ A. Albert, ${ }^{39}$ D. Duchardt,,${ }^{39}$ M. Erdmann, ${ }^{39}$ S. Erdweg, ${ }^{39}$ T. Esch, ${ }^{39}$ R. Fischer, ${ }^{39}$ S. Ghosh, ${ }^{39}$ A. Güth,${ }^{39}$ T. Hebbeker, ${ }^{39}$ C. Heidemann, ${ }^{39}$ K. Hoepfner, ${ }^{39}$ H. Keller, ${ }^{39}$ L. Mastrolorenzo, ${ }^{39}$ M. Merschmeyer, ${ }^{39}$ A. Meyer, ${ }^{39}$ P. Millet, ${ }^{39}$ S. Mukherjee, ${ }^{39}$ T. Pook, ${ }^{39}$ M. Radziej, ${ }^{39}$ H. Reithler, ${ }^{39}$ M. Rieger, ${ }^{39}$ A. Schmidt, ${ }^{39}$ D. Teyssier, ${ }^{39}$ S. Thüer, ${ }^{39}$ G. Flügge, ${ }^{40}$ O. Hlushchenko, ${ }^{40}$ T. Kress, ${ }^{40}$ A. Künsken, ${ }^{40}$ T. Müller, ${ }^{40}$ A. Nehrkorn, ${ }^{40}$ A. Nowack, ${ }^{40}$ C. Pistone, ${ }^{40}$ O. Pooth,${ }^{40}$ D. Roy, ${ }^{40}$ H. Sert, ${ }^{40}$ A. Stahl, ${ }^{40, r}$ M. Aldaya Martin, ${ }^{41}$ T. Arndt, ${ }^{41}$ C. Asawatangtrakuldee, ${ }^{41}$ I. Babounikau, ${ }^{41}$ K. Beernaert, ${ }^{41}$ O. Behnke, ${ }^{41}$ U. Behrens, ${ }^{41}$ A. Bermúdez Martínez, ${ }^{41}$ D. Bertsche, ${ }^{41}$ A. A. Bin Anuar, ${ }^{41}$ K. Borras, ${ }^{41, s}$ V. Botta, ${ }^{41}$ A. Campbell, ${ }^{41}$ P. Connor, ${ }^{41}$ C. Contreras-Campana, ${ }^{41}$ V. Danilov, ${ }^{41}$ A. De Wit,${ }^{41}$ M. M. Defranchis,${ }^{41}$ C. Diez Pardos,${ }^{41}$ D. Domínguez Damiani, ${ }^{41}$ G. Eckerlin, ${ }^{41}$ T. Eichhorn, ${ }^{41}$ A. Elwood, ${ }^{41}$ E. Eren, ${ }^{41}$ E. Gallo,${ }^{41, t}$ A. Geiser, ${ }^{41}$ A. Grohsjean, ${ }^{41}$ M. Guthoff, ${ }^{41}$ M. Haranko, ${ }^{41}$ A. Harb, ${ }^{41}$ J. Hauk, ${ }^{41}$ H. Jung, ${ }^{41}$ M. Kasemann, ${ }^{41}$ J. Keaveney, ${ }^{41}$ C. Kleinwort, ${ }^{41}$ J. Knolle, ${ }^{41}$ D. Krücker, ${ }^{41}$ W. Lange, ${ }^{41}$ A. Lelek, ${ }^{41}$ T. Lenz, ${ }^{41}$ J. Leonard, ${ }^{41}$ K. Lipka, ${ }^{41}$ W. Lohmann, ${ }^{41, u}$ R. Mankel, ${ }^{41}$ I.-A. Melzer-Pellmann, ${ }^{41}$ A. B. Meyer, ${ }^{41}$ M. Meyer, ${ }^{41}$ M. Missiroli, ${ }^{41}$ G. Mittag, ${ }^{41}$ J. Mnich,${ }^{41}$ V. Myronenko, ${ }^{41}$ S. K. Pflitsch, ${ }^{41}$ D. Pitzl, ${ }^{41}$ A. Raspereza, ${ }^{41}$ M. Savitskyi ${ }^{41}$ P. Saxena, ${ }^{41}$ P. Schütze, ${ }^{41}$ C. Schwanenberger ${ }^{41}$ R. Shevchenko, ${ }^{41}$ A. Singh, ${ }^{41}$ H. Tholen, ${ }^{41}$ O. Turkot,${ }^{41}$ A. Vagnerini, ${ }^{41}$ G. P. Van Onsem, ${ }^{41}$ R. Walsh, ${ }^{41}$ Y. Wen, ${ }^{41}$ K. Wichmann, ${ }^{41}$ C. Wissing, ${ }^{41}$ O. Zenaiev, ${ }^{41}$ R. Aggleton, ${ }^{42}$ S. Bein, ${ }^{42}$ L. Benato, ${ }^{42}$ A. Benecke, ${ }^{42}$ V. Blobel, ${ }^{42}$ T. Dreyer,${ }^{42}$ A. Ebrahimi, ${ }^{42}$ E. Garutti, ${ }^{42}$ D. Gonzalez, ${ }^{42}$ P. Gunnellini, ${ }^{42}$ J. Haller, ${ }^{42}$ A. Hinzmann, ${ }^{42}$ A. Karavdina, ${ }^{42}$ G. Kasieczka, ${ }^{42}$ R. Klanner, ${ }^{42}$ R. Kogler, ${ }^{42}$ N. Kovalchuk, ${ }^{42}$ S. Kurz, ${ }^{42}$ V. Kutzner, ${ }^{42}$ J. Lange, ${ }^{42}$ D. Marconi, ${ }^{42}$ J. Multhaup,${ }^{42}$ M. Niedziela, ${ }^{42}$ C. E. N. Niemeyer ${ }^{42}$ D. Nowatschin, ${ }^{42}$ A. Perieanu, ${ }^{42}$ A. Reimers, ${ }^{42}$ O. Rieger, ${ }^{42}$ C. Scharf ${ }^{42}$ P. Schleper, ${ }^{42}$ S. Schumann, ${ }^{42}$ J. Schwandt, ${ }^{42}$ J. Sonneveld, ${ }^{42}$ H. Stadie, ${ }^{42}$ G. Steinbrück, ${ }^{42}$ F. M. Stober, ${ }^{42}$ M. Stöver, ${ }^{42}$ A. Vanhoefer, ${ }^{42}$ B. Vormwald ${ }^{42}$ I. Zoi, ${ }^{42}$ M. Akbiyik, ${ }^{43}$ C. Barth, ${ }^{43}$ M. Baselga, ${ }^{43}$ S. Baur, ${ }^{43}$ E. Butz ${ }^{43}$ R. Caspart, ${ }^{43}$ T. Chwalek ${ }^{43}$ F. Colombo, ${ }^{43}$ W. De Boer, ${ }^{43}$ A. Dierlamm, ${ }^{43}$

K. El Morabit, ${ }^{43}$ N. Faltermann, ${ }^{43}$ B. Freund, ${ }^{43}$ M. Giffels, ${ }^{43}$ M. A. Harrendorf, ${ }^{43}$ F. Hartmann, ${ }^{43, r}$ S. M. Heindl, ${ }^{43}$ U. Husemann, ${ }^{43}$ F. Kassel, ${ }^{43, \mathrm{r}}$ I. Katkov, ${ }^{43, \mathrm{p}}$ S. Kudella, ${ }^{43}$ S. Mitra, ${ }^{43}$ M. U. Mozer, ${ }^{43}$ Th. Müller,${ }^{43}$ M. Plagge,${ }^{43}$ G. Quast,${ }^{43}$ K. Rabbertz, ${ }^{43}$ M. Schröder, ${ }^{43}$ I. Shvetsov,${ }^{43}$ G. Sieber, ${ }^{43}$ H. J. Simonis, ${ }^{43}$ R. Ulrich,${ }^{43}$ S. Wayand, ${ }^{43}$ M. Weber,${ }^{43}$ T. Weiler ${ }^{43}$ S. Williamson, ${ }^{43}$ C. Wöhrmann, ${ }^{43}$ R. Wolf, ${ }^{43}$ G. Anagnostou, ${ }^{44}$ G. Daskalakis, ${ }^{44}$ T. Geralis, ${ }^{44}$ A. Kyriakis, ${ }^{44}$ D. Loukas, ${ }^{44}$ G. Paspalaki, ${ }^{44}$ I. Topsis-Giotis, ${ }^{44}$ G. Karathanasis, ${ }^{45}$ S. Kesisoglou, ${ }^{45}$ P. Kontaxakis, ${ }^{45}$ A. Panagiotou, ${ }^{45}$ I. Papavergou, ${ }^{45}$ N. Saoulidou, ${ }^{45}$ E. Tziaferi, ${ }^{45}$ K. Vellidis, ${ }^{45}$ K. Kousouris, ${ }^{46}$ I. Papakrivopoulos, ${ }^{46}$ G. Tsipolitis, ${ }^{46}$ I. Evangelou, ${ }^{47}$ C. Foudas, ${ }^{47}$ P. Gianneios, ${ }^{47}$ P. Katsoulis,${ }^{47}$ P. Kokkas, ${ }^{47}$ S. Mallios, ${ }^{47}$ N. Manthos,${ }^{47}$ I. Papadopoulos,${ }^{47}$ E. Paradas,${ }^{47}$ J. Strologas, ${ }^{47}$ F. A. Triantis, ${ }^{47}$ D. Tsitsonis, ${ }^{47}$ M. Bartók, ${ }^{48, v}$ M. Csanad,${ }^{48}$ N. Filipovic, ${ }^{48}$ P. Major,${ }^{48}$ M. I. Nagy, ${ }^{48}$ G. Pasztor, ${ }^{48}$ O. Surányi,${ }^{48}$ G. I. Veres, ${ }^{48}$ G. Bencze, ${ }^{49}$ C. Hajdu, ${ }^{49}$ D. Horvath ${ }^{49, w}$ Á. Hunyadi, ${ }^{49}$ F. Sikler,${ }^{49}$ T. Á. Vámi,${ }^{49}$ V. Veszpremi, ${ }^{49}$ G. Vesztergombi, ${ }^{49, a, v}$ N. Beni, ${ }^{50}$ S. Czellar, ${ }^{50}$ J. Karancsi,${ }^{50, x}$ A. Makovec,${ }^{50}$ J. Molnar, ${ }^{50}$ Z. Szillasi, ${ }^{50}$ P. Raics, ${ }^{51}$ Z. L. Trocsanyi, ${ }^{51}$ B. Ujvari, ${ }^{51}$ S. Choudhury, ${ }^{52}$ J. R. Komaragiri, ${ }^{52}$ P. C. Tiwari, ${ }^{52}$ S. Bahinipati, ${ }^{53, y}$ C. Kar, ${ }^{53}$ P. Mal, ${ }^{53}$ K. Mandal, ${ }^{53}$ A. Nayak, ${ }^{53, z}$ D. K. Sahoo, ${ }^{53, y}$ S. K. Swain, ${ }^{53}$ S. Bansal, ${ }^{54}$ S. B. Beri, ${ }^{54}$ V. Bhatnagar,${ }^{54}$ S. Chauhan, ${ }^{54}$ R. Chawla ${ }^{54}$ N. Dhingra, ${ }^{54}$ R. Gupta, ${ }^{54}$ A. Kaur, ${ }^{54}$ M. Kaur, ${ }^{54}$ S. Kaur,${ }^{54}$ R. Kumar, ${ }^{54}$ P. Kumari, ${ }^{54}$ M. Lohan, ${ }^{54}$ A. Mehta,${ }^{54}$ K. Sandeep, ${ }^{54}$ S. Sharma, ${ }^{54}$ J. B. Singh ${ }^{54}$ A. K. Virdi, ${ }^{54}$ G. Walia, ${ }^{54}$ A. Bhardwaj, ${ }^{55}$ B. C. Choudhary, ${ }^{55}$ R. B. Garg ${ }^{55}$ M. Gola, ${ }^{55}$ S. Keshri, ${ }^{55}$ Ashok Kumar, ${ }^{55}$ S. Malhotra, ${ }^{55}$ M. Naimuddin, ${ }^{55}$ P. Priyanka, ${ }^{55}$ K. Ranjan, ${ }^{55}$ Aashaq Shah, ${ }^{55}$ R. Sharma, ${ }^{55}$ R. Bhardwaj, ${ }^{56, a a}$ M. Bharti, ${ }^{56, a a}$ R. Bhattacharya,${ }^{56}$ S. Bhattacharya, ${ }^{56}$ U. Bhawandeep, ${ }^{56, \text { aa }}$ D. Bhowmik, ${ }^{56}$ S. Dey, ${ }^{56}$ S. Dutt, ${ }^{56 \text {,aa }}$ S. Dutta, ${ }^{56}$ S. Ghosh,${ }^{56}$ K. Mondal, ${ }^{56}$ S. Nandan, ${ }^{56}$ A. Purohit, ${ }^{56}$ P. K. Rout, ${ }^{56}$ A. Roy, ${ }^{56}$

S. Roy Chowdhury, ${ }^{56}$ G. Saha, ${ }^{56}$ S. Sarkar, ${ }^{56}$ M. Sharan, ${ }^{56}$ B. Singh, ${ }^{56, a a}$ S. Thakur, ${ }^{56, a a}$ P. K. Behera ${ }^{57}$ R. Chudasama ${ }^{58}$ D. Dutta, ${ }^{58}$ V. Jha, ${ }^{58}$ V. Kumar, ${ }^{58}$ P. K. Netrakanti, ${ }^{58}$ L. M. Pant, ${ }^{58}$ P. Shukla, ${ }^{58}$ T. Aziz, ${ }^{59}$ M. A. Bhat,${ }^{59}$ S. Dugad, ${ }^{59}$ G. B. Mohanty, ${ }^{59}$ N. Sur,${ }^{59}$ B. Sutar, ${ }^{59}$ Ravindra Kumar Verma, ${ }^{59}$ S. Banerjee, ${ }^{60}$ S. Bhattacharya,${ }^{60}$ S. Chatterjee, ${ }^{60}$ P. Das,${ }^{60}$ M. Guchait, ${ }^{60}$ Sa. Jain, ${ }^{60}$ S. Karmakar, ${ }^{60}$ S. Kumar, ${ }^{60}$ M. Maity, ${ }^{60, b b}$ G. Majumder, ${ }^{60}$ K. Mazumdar, ${ }^{60}$ N. Sahoo, ${ }^{60}$ T. Sarkar, ${ }^{60, b b}$ S. Chauhan, ${ }^{61}$ S. Dube ${ }^{61}$ V. Hegde ${ }^{61}$ A. Kapoor, ${ }^{61}$ K. Kothekar, ${ }^{61}$ S. Pandey, ${ }^{61}$ A. Rane, ${ }^{61}$ S. Sharma, ${ }^{61}$ S. Chenarani, ${ }^{62, c c}$ E. Eskandari Tadavani, ${ }^{62}$ S. M. Etesami, ${ }^{62, c c}$ M. Khakzad, ${ }^{62}$ M. Mohammadi Najafabadi, ${ }^{62}$ M. Naseri, ${ }^{62}$ 
F. Rezaei Hosseinabadi ${ }^{62}$ B. Safarzadeh, ${ }^{62, d d}$ M. Zeinali, ${ }^{62}$ M. Felcini,${ }^{63}$ M. Grunewald ${ }^{63}$ M. Abbrescia,${ }^{64 a, 64 b}$ C. Calabria ${ }^{64 a, 64 b}$ A. Colaleo, ${ }^{64 a}$ D. Creanza, ${ }^{64 a, 64 c}$ L. Cristella, ${ }^{64 a, 64 b}$ N. De Filippis, ${ }^{64 a, 64 c}$ M. De Palma, ${ }^{64 a, 64 b}$ A. Di Florio, ${ }^{64 a, 64 b}$ F. Errico, ${ }^{64 a, 64 b}$ L. Fiore, ${ }^{64 a}$ A. Gelmi, ${ }^{64 a, 64 b}$ G. Iaselli, ${ }^{64 a, 64 c}$ M. Ince, ${ }^{64 a, 64 b}$ S. Lezki, ${ }^{64 a, 64 b}$ G. Maggi, ${ }^{64 a, 64 c}$ M. Maggi, ${ }^{64 a}$ G. Miniello, ${ }^{64 a, 64 b}$ S. My, ${ }^{64 a, 64 b}$ S. Nuzzo, ${ }^{64 a, 64 b}$ A. Pompili, ${ }^{64 a, 64 b}$ G. Pugliese, ${ }^{64 a, 64 c}$ R. Radogna, ${ }^{64 a}$ A. Ranieri, ${ }^{64 a}$ G. Selvaggi, ${ }^{64,64 b}$ A. Sharma ${ }^{64 a}$ L. Silvestris, ${ }^{64 a}$ R. Venditti, ${ }^{64 a}$ P. Verwilligen, ${ }^{64 a}$ G. Zito, ${ }^{64 a}$ G. Abbiendi, ${ }^{65 a}$ C. Battilana ${ }^{65 a, 65 b}$ D. Bonacorsi ${ }^{65 a, 65 b}$ L. Borgonovi ${ }^{65 a, 65 b}$ S. Braibant-Giacomelli, ${ }^{65 a, 65 b}$ R. Campanini, ${ }^{65 a, 65 b}$ P. Capiluppi ${ }^{65 a, 65 b}$ A. Castro, ${ }^{65 a, 65 b}$ F. R. Cavallo, ${ }^{65 a}$ S. S. Chhibra,${ }^{65 a, 65 b}$ C. Ciocca, ${ }^{65 a}$ G. Codispoti, ${ }^{65 a, 65 b}$ M. Cuffiani ${ }^{65 a, 65 b}$ G. M. Dallavalle, ${ }^{65 a}$ F. Fabbri, ${ }^{65 a}$ A. Fanfani, ${ }^{65 a, 65 b}$ E. Fontanesi ${ }^{65 a}$ P. Giacomelli, ${ }^{65 a}$ C. Grandi, ${ }^{65 a}$ L. Guiducci, ${ }^{65 a, 65 b}$ S. Lo Meo, ${ }^{65 a}$ S. Marcellini, ${ }^{65 a}$ G. Masetti, ${ }^{65 a}$ A. Montanari, ${ }^{65 a}$ F. L. Navarria, ${ }^{65 a, 65 b}$ A. Perrotta, ${ }^{65 a}$ F. Primavera, ${ }^{65 a, 65 b, r}$ A. M. Rossi, ${ }^{65 a, 65 b}$ T. Rovelli, ${ }^{65 a, 65 b}$ G. P. Siroli, ${ }^{65 a, 65 b}$ N. Tosi, ${ }^{65 a}$ S. Albergo, ${ }^{66 a, 66 b}$ A. Di Mattia, ${ }^{66 a}$ R. Potenza, ${ }^{66 a, 66 b}$ A. Tricomi ${ }^{66 a, 66 b}$ C. Tuve, ${ }^{66 a, 66 b}$ G. Barbagli, ${ }^{67 a}$ K. Chatterjee, ${ }^{67 a, 67 b}$ V. Ciulli, ${ }^{67 a, 67 b}$ C. Civinini, ${ }^{67 a}$ R. D’Alessandro, ${ }^{67 a, 67 b}$ E. Focardi, ${ }^{67,67 b}$ G. Latino, ${ }^{67 a}$ P. Lenzi, ${ }^{67 a, 67 b}$ M. Meschini,${ }^{67 a}$ S. Paoletti ${ }^{67 a}$ L. Russo, ${ }^{67 a, e e}$ G. Sguazzoni, ${ }^{67 a}$ D. Strom, ${ }^{67 a}$ L. Viliani, ${ }^{67 \mathrm{a}}$ L. Benussi, ${ }^{68}$ S. Bianco,${ }^{68}$ F. Fabbri,${ }^{68}$ D. Piccolo, ${ }^{68}$ F. Ferro ${ }^{69 a}$ F. Ravera, ${ }^{69 a, 69 b}$ E. Robutti, ${ }^{69 a}$ S. Tosi,${ }^{69 a, 69 b}$ A. Benaglia, ${ }^{70 a}$ A. Beschi, ${ }^{70 a, 70 b}$ F. Brivio, ${ }^{70 a, 70 b}$ V. Ciriolo, ${ }^{70 a, 70 b, r}$ S. Di Guida, ${ }^{70 a, 70 b, r}$ M. E. Dinardo,${ }^{70 a, 70 b}$ S. Fiorendi, ${ }^{70 a, 70 b}$ S. Gennai ${ }^{70 a}$ A. Ghezzi, ${ }^{70 a, 70 b}$ P. Govoni, ${ }^{70 a, 70 b}$ M. Malberti, ${ }^{70 a, 70 b}$ S. Malvezzi, ${ }^{70 a}$ A. Massironi,${ }^{70 a, 70 b}$ D. Menasce, ${ }^{70 a}$ F. Monti, ${ }^{70 a}$ L. Moroni, ${ }^{70 a}$ M. Paganoni, ${ }^{70,70 b}$ D. Pedrini, ${ }^{70 a}$ S. Ragazzi, ${ }^{70 a, 70 b}$ T. Tabarelli de Fatis, ${ }^{70 a, 70 b}$ D. Zuolo, ${ }^{70 a, 70 b}$ S. Buontempo ${ }^{71 \mathrm{a}}$ N. Cavallo, ${ }^{71 a, 71 \mathrm{c}}$ A. De Iorio ${ }^{71 \mathrm{a}, 71 \mathrm{~b}}$ A. Di Crescenzo,${ }^{71 \mathrm{a}, 7 \mathrm{~b}}$ F. Fabozzi, ${ }^{71 \mathrm{a}, 71 \mathrm{c}}$ F. Fienga, ${ }^{71 \mathrm{a}}$ G. Galati, ${ }^{71 \mathrm{a}}$ A. O. M. Iorio, ${ }^{71 a, 71 b}$ W. A. Khan, ${ }^{71 a}$ L. Lista, ${ }^{71 a}$ S. Meola,${ }^{71 a, 71 d, r}$ P. Paolucci, ${ }^{71 a, r}$ C. Sciacca, ${ }^{71 a, 71 b}$ E. Voevodina, ${ }^{71 a, 71 b}$ P. Azzi, ${ }^{72 a}$ N. Bacchetta, ${ }^{72 a}$ D. Bisello, ${ }^{72 a, 72 b}$ A. Boletti, ${ }^{72 a, 72 b}$ A. Bragagnolo, ${ }^{72 a}$ R. Carlin, ${ }^{72 a, 72 b}$ P. Checchia, ${ }^{72 a}$ M. Dall'Osso, ${ }^{72 a, 72 b}$ P. De Castro Manzano, ${ }^{72 a}$ T. Dorigo, ${ }^{72 a}$ U. Dosselli, ${ }^{72 a}$ F. Gasparini, ${ }^{72 a, 72 b}$ U. Gasparini, ${ }^{72 a, 72 b}$ A. Gozzelino, ${ }^{72 a}$ S. Y. Hoh, ${ }^{72 a}$ S. Lacaprara, ${ }^{72 a}$ P. Lujan, ${ }^{72 a}$ M. Margoni, ${ }^{72 a, 72 b}$ A. T. Meneguzzo, ${ }^{72 a, 72 b}$ J. Pazzini, ${ }^{72 a, 72 b}$ P. Ronchese, ${ }^{72 a, 72 b}$ R. Rossin, ${ }^{72 a, 72 b}$ F. Simonetto, ${ }^{72 a, 72 b}$ A. Tiko, ${ }^{72 a}$ E. Torassa, ${ }^{72 a}$ M. Zanetti, ${ }^{72 a, 72 b}$ P. Zotto, ${ }^{72 a, 72 b}$ G. Zumerle, ${ }^{72 a, 72 b}$ A. Braghieri, ${ }^{73 a}$ A. Magnani, ${ }^{73 a}$ P. Montagna, ${ }^{73 a, 73 b}$ S. P. Ratti ${ }^{73 a, 73 b}$ V. Re,${ }^{73 a}$ M. Ressegotti, ${ }^{73 a, 73 b}$ C. Riccardi, ${ }^{73 a, 73 b}$ P. Salvini, ${ }^{73 a}$ I. Vai, ${ }^{73 a, 73 b}$ P. Vitulo, ${ }^{73 a, 73 b}$ M. Biasini, ${ }^{74 a, 74 b}$ G. M. Bilei, ${ }^{74 a}$ C. Cecchi, ${ }^{74 a, 74 b}$ D. Ciangottini, ${ }^{74 a, 74 b}$ L. Fanò, ${ }^{74 a, 74 b}$ P. Lariccia, ${ }^{74 a, 74 b}$ R. Leonardi, ${ }^{74 a, 74 b}$ E. Manoni, ${ }^{74 a}$ G. Mantovani, ${ }^{74 a, 74 b}$ V. Mariani, ${ }^{74 a, 74 b}$ M. Menichelli, ${ }^{74 a}$ A. Rossi, ${ }^{74 a, 74 b}$ A. Santocchia,${ }^{74 a, 74 b}$ D. Spiga, ${ }^{74 a}$ K. Androsov, ${ }^{75 a}$ P. Azzurri, ${ }^{75 a}$ G. Bagliesi, ${ }^{75 a}$ L. Bianchini, ${ }^{75 a}$ T. Boccali, ${ }^{75 a}$ L. Borrello, ${ }^{75 a}$ R. Castaldi, ${ }^{75 a}$ M. A. Ciocci, ${ }^{75 a, 75 b}$ R. Dell'Orso, ${ }^{75 a}$ G. Fedi, ${ }^{75 a}$ F. Fiori, ${ }^{75 a, 75 c}$ L. Giannini, ${ }^{75 a, 75 c}$ A. Giassi, ${ }^{75 a}$ M. T. Grippo, ${ }^{75 a}$ F. Ligabue,${ }^{75 a, 75 c}$ E. Manca ${ }^{75 a, 75 c}$ G. Mandorli, ${ }^{75 a, 75 c}$ A. Messineo, ${ }^{75 a, 75 b}$ F. Palla, ${ }^{75 a}$ A. Rizzi,${ }^{75 a, 75 b}$ P. Spagnolo, ${ }^{75 a}$ R. Tenchini, ${ }^{75 a}$ G. Tonelli, ${ }^{75 a, 75 b}$ A. Venturi, ${ }^{75 a}$ P. G. Verdini, ${ }^{75 a}$ L. Barone ${ }^{76 a, 76 b}$ F. Cavallari, ${ }^{76 a}$ M. Cipriani, ${ }^{76 a, 76 b}$ D. Del Re,${ }^{76 a, 76 b}$ E. Di Marco, ${ }^{76 a, 76 b}$ M. Diemoz ${ }^{76 a}$ S. Gelli, ${ }^{76 a, 76 b}$ E. Longo, ${ }^{76 a, 76 b}$ B. Marzocchi, ${ }^{76 a, 76 b}$ P. Meridiani, ${ }^{76 a}$ G. Organtini, ${ }^{76 a, 76 b}$ F. Pandolfi, ${ }^{76 a}$ R. Paramatti ${ }^{76 a, 76 b}$ F. Preiato, ${ }^{76 a, 76 b}$ S. Rahatlou, ${ }^{76 a, 76 b}$ C. Rovelli, ${ }^{76 a}$ F. Santanastasio, ${ }^{76 a, 76 b}$ N. Amapane, ${ }^{77 a, 77 b}$ R. Arcidiacono, ${ }^{77,77 c}$ S. Argiro, ${ }^{77 a, 77 b}$ M. Arneodo, ${ }^{77 a, 77 c}$ N. Bartosik, ${ }^{77 a}$ R. Bellan, ${ }^{77 a, 77 b}$ C. Biino, ${ }^{77 a}$ N. Cartiglia, ${ }^{77 a}$ F. Cenna, ${ }^{77 a, 77 b}$ S. Cometti, ${ }^{77 a}$ M. Costa $,{ }^{77 a}, 77 b$ R. Covarelli, ${ }^{77 a, 77 b}$ N. Demaria, ${ }^{77 a}$ B. Kiani, ${ }^{77 a, 77 b}$ C. Mariotti, ${ }^{77 a}$ S. Maselli, ${ }^{77 a}$ E. Migliore, ${ }^{77 a, 77 b}$ V. Monaco, ${ }^{77 a, 77 b}$ E. Monteil,${ }^{77 a, 77 b}$ M. Monteno, ${ }^{77 a}$ M. M. Obertino, ${ }^{77 a, 77 b}$ L. Pacher,${ }^{77 a, 77 b}$ N. Pastrone ${ }^{77 a}$ M. Pelliccioni, ${ }^{77 a}$ G. L. Pinna Angioni, ${ }^{77 a, 77 b}$ A. Romero, ${ }^{77 a, 77 b}$ M. Ruspa, ${ }^{77 a, 77 c}$ R. Sacchi, ${ }^{77 a, 77 b}$ K. Shchelina, ${ }^{77 a, 77 b}$ V. Sola, ${ }^{77 a}$ A. Solano, ${ }^{77 a, 77 b}$ D. Soldi, ${ }^{77,77 b}$ A. Staiano, ${ }^{77 a}$ S. Belforte, ${ }^{78 a}$ V. Candelise ${ }^{78 a, 78 b}$ M. Casarsa, ${ }^{78 a}$ F. Cossutti, ${ }^{78 a}$ A. Da Rold ${ }^{78 a, 78 b}$ G. Della Ricca, ${ }^{78 a, 78 b}$ F. Vazzoler, ${ }^{78 a}, 78 b$ A. Zanetti, ${ }^{78 a}$ D. H. Kim, ${ }^{79}$ G. N. Kim, ${ }^{79}$ M. S. Kim, ${ }^{79}$ J. Lee, ${ }^{79}$ S. Lee, ${ }^{79}$ S. W. Lee, ${ }^{79}$ C. S. Moon, ${ }^{79}$ Y. D. Oh ${ }^{79}$ S. I. Pak,${ }^{79}$ S. Sekmen, ${ }^{79}$ D. C. Son, ${ }^{79}$ Y. C. Yang ${ }^{79}$ H. Kim, ${ }^{80}$ D. H. Moon,${ }^{80}$ G. Oh, ${ }^{80}$ B. Francois,${ }^{81}$ J. Goh, ${ }^{81, f f}$ T. J. Kim, ${ }^{81}$ S. Cho, ${ }^{82}$ S. Choi, ${ }^{82}$ Y. Go,${ }^{82}$ D. Gyun, ${ }^{82}$ S. Ha ${ }^{82}$ B. Hong, ${ }^{82}$ Y. Jo, ${ }^{82}$ K. Lee, ${ }^{82}$ K. S. Lee, ${ }^{82}$ S. Lee, ${ }^{82}$ J. Lim, ${ }^{82}$ S. K. Park, ${ }^{82}$ Y. Roh ${ }^{82}$ H. S. Kim, ${ }^{83}$ J. Almond ${ }^{84}$ J. Kim, ${ }^{84}$ J. S. Kim ${ }^{84}$ H. Lee, ${ }^{84}$ K. Lee, ${ }^{84}$ K. Nam, ${ }^{84}$ S. B. Oh, ${ }^{84}$ B. C. Radburn-Smith, ${ }^{84}$ S. h. Seo, ${ }^{84}$ U. K. Yang, ${ }^{84}$ H. D. Yoo, ${ }^{84}$ G. B. Yu, ${ }^{84}$ D. Jeon, ${ }^{85}$ H. Kim, ${ }^{85}$ J. H. Kim,${ }^{85}$ J. S. H. Lee, ${ }^{85}$ I. C. Park, ${ }^{85}$ Y. Choi, ${ }^{86}$ C. Hwang, ${ }^{86}$ J. Lee, ${ }^{86}$ I. Yu, ${ }^{86}$ V. Dudenas, ${ }^{87}$ A. Juodagalvis, ${ }^{87}$ J. Vaitkus, ${ }^{87}$ I. Ahmed, ${ }^{88}$ Z. A. Ibrahim, ${ }^{88}$ M. A. B. Md Ali, ${ }^{88, g g}$ F. Mohamad Idris, ${ }^{88, \text { hh }}$ W. A. T. Wan Abdullah, ${ }^{88}$ M. N. Yusli, ${ }^{88}$ Z. Zolkapli, ${ }^{88}$ J. F. Benitez, ${ }^{89}$ A. Castaneda Hernandez, ${ }^{89}$ J. A. Murillo Quijada, ${ }^{89}$ H. Castilla-Valdez, ${ }^{90}$ E. De La Cruz-Burelo, ${ }^{90}$ M. C. Duran-Osuna, ${ }^{90}$ I. Heredia-De La Cruz, ${ }^{90, \text { ii }}$ R. Lopez-Fernandez,${ }^{90}$ J. Mejia Guisao, ${ }^{90}$ R. I. Rabadan-Trejo, ${ }^{90}$ M. Ramirez-Garcia, ${ }^{90}$ G. Ramirez-Sanchez,${ }^{90}$ R Reyes-Almanza, ${ }^{90}$ A. Sanchez-Hernandez, ${ }^{90}$ S. Carrillo Moreno, ${ }^{91}$ C. Oropeza Barrera, ${ }^{91}$ F. Vazquez Valencia, ${ }^{91}$ J. Eysermans, ${ }^{92}$ I. Pedraza, ${ }^{92}$ H. A. Salazar Ibarguen, ${ }^{92}$ C. Uribe Estrada, ${ }^{92}$ A. Morelos Pineda, ${ }^{93}$ D. Krofcheck, ${ }^{94}$ S. Bheesette, ${ }^{95}$ P. H. Butler, ${ }^{95}$ A. Ahmad, ${ }^{96}$ M. Ahmad, ${ }^{96}$ M. I. Asghar, ${ }^{96}$ Q. Hassan, ${ }^{96}$ 
H. R. Hoorani ${ }^{96}$ A. Saddique, ${ }^{96}$ M. A. Shah,${ }^{96}$ M. Shoaib,${ }^{96}$ M. Waqas, ${ }^{96}$ H. Bialkowska, ${ }^{97}$ M. Bluj,${ }^{97}$ B. Boimska,${ }^{97}$ T. Frueboes, ${ }^{97}$ M. Górski, ${ }^{97}$ M. Kazana,${ }^{97}$ M. Szleper,${ }^{97}$ P. Traczyk, ${ }^{97}$ P. Zalewski,${ }^{97}$ K. Bunkowski, ${ }^{98}$ A. Byszuk,${ }^{98, j j}$ K. Doroba, ${ }^{98}$ A. Kalinowski, ${ }^{98}$ M. Konecki, ${ }^{98}$ J. Krolikowski, ${ }^{98}$ M. Misiura, ${ }^{98}$ M. Olszewski, ${ }^{98}$ A. Pyskir, ${ }^{98}$ M. Walczak, ${ }^{98}$ M. Araujo, ${ }^{99}$ P. Bargassa, ${ }^{99}$ C. Beirão Da Cruz E Silva, ${ }^{99}$ A. Di Francesco, ${ }^{99}$ P. Faccioli, ${ }^{99}$ B. Galinhas, ${ }^{99}$ M. Gallinaro, ${ }^{99}$ J. Hollar, ${ }^{99}$ N. Leonardo,${ }^{99}$ M. V. Nemallapudi, ${ }^{99}$ J. Seixas, ${ }^{99}$ G. Strong, ${ }^{99}$ O. Toldaiev, ${ }^{99}$ D. Vadruccio, ${ }^{99}$ J. Varela, ${ }^{99}$ S. Afanasiev, ${ }^{100}$ P. Bunin, ${ }^{100}$ M. Gavrilenko, ${ }^{100}$ I. Golutvin, ${ }^{100}$ I. Gorbunov, ${ }^{100}$ A. Kamenev, ${ }^{100}$ V. Karjavine, ${ }^{100}$ A. Lanev, ${ }^{100}$

A. Malakhov, ${ }^{100}$ V. Matveev, ${ }^{100, k k, 11}$ P. Moisenz, ${ }^{100}$ V. Palichik, ${ }^{100}$ V. Perelygin, ${ }^{100}$ S. Shmatov, ${ }^{100}$ S. Shulha, ${ }^{100}$ N. Skatchkov, ${ }^{100}$ V. Smirnov, ${ }^{100}$ N. Voytishin, ${ }^{100}$ A. Zarubin, ${ }^{100}$ V. Golovtsov, ${ }^{101}$ Y. Ivanov, ${ }^{101}$ V. Kim, ${ }^{101, m m}$ E. Kuznetsova, ${ }^{101, \text { nn }}$ P. Levchenko, ${ }^{101}$ V. Murzin, ${ }^{101}$ V. Oreshkin,,${ }^{101}$ I. Smirnov, ${ }^{101}$ D. Sosnov, ${ }^{101}$ V. Sulimov, ${ }^{101}$ L. Uvarov, ${ }^{101}$ S. Vavilov, ${ }^{101}$ A. Vorobyev, ${ }^{101}$ Yu. Andreev, ${ }^{102}$ A. Dermenev, ${ }^{102}$ S. Gninenko, ${ }^{102}$ N. Golubev, ${ }^{102}$ A. Karneyeu, ${ }^{102}$ M. Kirsanov, ${ }^{102}$ N. Krasnikov, ${ }^{102}$ A. Pashenkov, ${ }^{102}$ D. Tlisov, ${ }^{102}$ A. Toropin, ${ }^{102}$ V. Epshteyn, ${ }^{103}$ V. Gavrilov, ${ }^{103}$ N. Lychkovskaya, ${ }^{103}$ V. Popov, ${ }^{103}$ I. Pozdnyakov, ${ }^{103}$ G. Safronov, ${ }^{103}$ A. Spiridonov, ${ }^{103}$ A. Stepennov, ${ }^{103}$

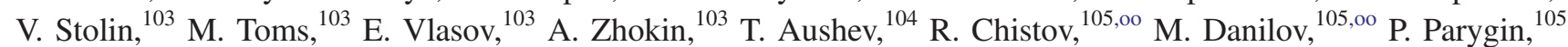
D. Philippov, ${ }^{105}$ S. Polikarpov, ${ }^{105,00}$ E. Tarkovskii, ${ }^{105}$ V. Andreev, ${ }^{106}$ M. Azarkin, ${ }^{106}$ I. Dremin, ${ }^{106,11}$ M. Kirakosyan, ${ }^{106}$ S. V. Rusakov, ${ }^{106}$ A. Terkulov, ${ }^{106}$ A. Baskakov, ${ }^{107}$ A. Belyaev, ${ }^{107}$ E. Boos, ${ }^{107}$ V. Bunichev, ${ }^{107}$ M. Dubinin, ${ }^{107, p p}$ L. Dudko, ${ }^{107}$ A. Ershov, ${ }^{107}$ A. Gribushin, ${ }^{107}$ V. Klyukhin, ${ }^{107}$ I. Lokhtin, ${ }^{107}$ I. Miagkov,${ }^{107}$ S. Obraztsov, ${ }^{107}$ M. Perfilov, ${ }^{107}$ S. Petrushanko, ${ }^{107}$ V. Savrin, ${ }^{107}$ A. Barnyakov, ${ }^{108, q q}$ V. Blinov, ${ }^{108, q q}$ T. Dimova, ${ }^{108, q q}$ L. Kardapoltsev, ${ }^{108, q q}$ Y. Skovpen, ${ }^{108, q q}$ I. Azhgirey, ${ }^{109}$ I. Bayshev, ${ }^{109}$ S. Bitioukov, ${ }^{109}$ D. Elumakhov, ${ }^{109}$ A. Godizov, ${ }^{109}$ V. Kachanov, ${ }^{109}$ A. Kalinin, ${ }^{109}$ D. Konstantinov, ${ }^{109}$ P. Mandrik, ${ }^{109}$ V. Petrov ${ }^{109}$ R. Ryutin, ${ }^{109}$ S. Slabospitskii, ${ }^{109}$ A. Sobol, ${ }^{109}$ S. Troshin, ${ }^{109}$ N. Tyurin, ${ }^{109}$ A. Uzunian, ${ }^{109}$ A. Volkov, ${ }^{109}$ A. Babaev, ${ }^{110}$ S. Baidali, ${ }^{110}$ V. Okhotnikov, ${ }^{110}$ P. Adzic, ${ }^{111, \text { rr }}$ P. Cirkovic,,${ }^{111}$ D. Devetak, ${ }^{111}$ M. Dordevic, ${ }^{111}$ J. Milosevic, ${ }^{111}$ J. Alcaraz Maestre, ${ }^{112}$ A. Álvarez Fernández, ${ }^{112}$ I. Bachiller, ${ }^{112}$ M. Barrio Luna, ${ }^{112}$ J. A. Brochero Cifuentes, ${ }^{112}$ M. Cerrada ${ }^{112}$ N. Colino, ${ }^{112}$ B. De La Cruz,${ }^{112}$ A. Delgado Peris ${ }^{112}$ C. Fernandez Bedoya, ${ }^{112}$ J. P. Fernández Ramos, ${ }^{112}$ J. Flix, ${ }^{112}$ M. C. Fouz, ${ }^{112}$ O. Gonzalez Lopez, ${ }^{112}$ S. Goy Lopez, ${ }^{112}$ J. M. Hernandez, ${ }^{112}$ M. I. Josa, ${ }^{112}$ D. Moran,${ }^{112}$ A. Pérez-Calero Yzquierdo, ${ }^{112}$ J. Puerta Pelayo,${ }^{112}$ I. Redondo, ${ }^{112}$ L. Romero, ${ }^{112}$ M. S. Soares, ${ }^{112}$ A. Triossi, ${ }^{112}$ C. Albajar, ${ }^{113}$ J. F. de Trocóniz, ${ }^{113}$ J. Cuevas, ${ }^{114}$ C. Erice,${ }^{114}$ J. Fernandez Menendez,${ }^{114}$ S. Folgueras, ${ }^{114}$ I. Gonzalez Caballero, ${ }^{114}$ J. R. González Fernández, ${ }^{114}$ E. Palencia Cortezon, ${ }^{114}$ V. Rodríguez Bouza, ${ }^{114}$ S. Sanchez Cruz, ${ }^{114}$ P. Vischia, ${ }^{114}$ J. M. Vizan Garcia, ${ }^{114}$ I. J. Cabrillo, ${ }^{115}$ A. Calderon, ${ }^{115}$ B. Chazin Quero, ${ }^{115}$ J. Duarte Campderros, ${ }^{115}$ M. Fernandez, ${ }^{115}$ P. J. Fernández Manteca, ${ }^{115}$ A. García Alonso, ${ }^{115}$ J. Garcia-Ferrero, ${ }^{115}$ G. Gomez, ${ }^{115}$ A. Lopez Virto, ${ }^{115}$ J. Marco, ${ }^{115}$ C. Martinez Rivero, ${ }^{115}$ P. Martinez Ruiz del Arbol, ${ }^{115}$ F. Matorras, ${ }^{115}$ J. Piedra Gomez, ${ }^{115}$ C. Prieels, ${ }^{115}$ T. Rodrigo, ${ }^{115}$ A. Ruiz-Jimeno, ${ }^{115}$ L. Scodellaro, ${ }^{115}$ N. Trevisani, ${ }^{115}$ I. Vila, ${ }^{115}$ R. Vilar Cortabitarte, ${ }^{115}$ N. Wickramage, ${ }^{116}$ D. Abbaneo, ${ }^{117}$ B. Akgun, ${ }^{117}$ E. Auffray, ${ }^{117}$ G. Auzinger, ${ }^{117}$ P. Baillon, ${ }^{117}$ A. H. Ball, ${ }^{117}$ D. Barney, ${ }^{117}$ J. Bendavid, ${ }^{117}$ M. Bianco ${ }^{117}$ A. Bocci, ${ }^{117}$ C. Botta, ${ }^{117}$ E. Brondolin, ${ }^{117}$ T. Camporesi, ${ }^{117}$ M. Cepeda,${ }^{117}$ G. Cerminara,${ }^{117}$ E. Chapon, ${ }^{117}$ Y. Chen ${ }^{117}$ G. Cucciati, ${ }^{117}$ D. d'Enterria, ${ }^{117}$ A. Dabrowski,${ }^{117}$ N. Daci, ${ }^{117}$ V. Daponte, ${ }^{117}$ A. David, ${ }^{117}$ A. De Roeck, ${ }^{117}$ N. Deelen, ${ }^{117}$ M. Dobson, ${ }^{117}$ M. Dünser ${ }^{117}$ N. Dupont, ${ }^{117}$ A. Elliott-Peisert, ${ }^{117}$ P. Everaerts, ${ }^{117}$ F. Fallavollita, ${ }^{117, s s}$ D. Fasanella, ${ }^{117}$ G. Franzoni, ${ }^{117}$ J. Fulcher, ${ }^{117}$ W. Funk, ${ }^{117}$ D. Gigi, ${ }^{117}$ A. Gilbert, ${ }^{117}$ K. Gill, ${ }^{117}$ F. Glege, ${ }^{117}$ M. Guilbaud, ${ }^{117}$ D. Gulhan, ${ }^{117}$ J. Hegeman, ${ }^{117}$ C. Heidegger, ${ }^{117}$ V. Innocente,${ }^{117}$ A. Jafari, ${ }^{117}$ P. Janot, ${ }^{117}$ O. Karacheban, ${ }^{117, u}$ J. Kieseler, ${ }^{117}$ A. Kornmayer, ${ }^{117}$ M. Krammer, ${ }^{117, b}$ C. Lange,${ }^{117}$ P. Lecoq,${ }^{117}$ C. Lourenço, ${ }^{117}$ L. Malgeri, ${ }^{117}$ M. Mannelli, ${ }^{117}$ F. Meijers, ${ }^{117}$ J. A. Merlin, ${ }^{117}$ S. Mersi ${ }^{117}$ E. Meschi,${ }^{117}$ P. Milenovic, ${ }^{117, t t}$ F. Moortgat,${ }^{117}$ M. Mulders, ${ }^{117}$ J. Ngadiuba,${ }^{117}$ S. Nourbakhsh ${ }^{117}$ S. Orfanelli ${ }^{117}$ L. Orsini ${ }^{117}$ F. Pantaleo, ${ }^{117, r}$ L. Pape, ${ }^{117}$ E. Perez,${ }^{117}$ M. Peruzzi, ${ }^{117}$ A. Petrilli, ${ }^{117}$ G. Petrucciani, ${ }^{117}$ A. Pfeiffer ${ }^{117}$ M. Pierini,${ }^{117}$ F. M. Pitters, ${ }^{117}$ D. Rabady, ${ }^{117}$ A. Racz, ${ }^{117}$ T. Reis, ${ }^{117}$ G. Rolandi, ${ }^{117, \text { uu }}$ M. Rovere, ${ }^{117}$ H. Sakulin, ${ }^{117}$ C. Schäfer, ${ }^{117}$ C. Schwick, ${ }^{117}$ M. Seidel, ${ }^{117}$ M. Selvaggi, ${ }^{117}$ A. Sharma, ${ }^{117}$ P. Silva, ${ }^{117}$ P. Sphicas, ${ }^{117, v v}$ A. Stakia, ${ }^{117}$ J. Steggemann, ${ }^{117}$ M. Tosi, ${ }^{117}$ D. Treille, ${ }^{117}$ A. Tsirou, ${ }^{117}$ V. Veckalns, ${ }^{117, w w}$ M. Verzetti, ${ }^{117}$ W. D. Zeuner, ${ }^{117}$ L. Caminada, ${ }^{118, x x}$ K. Deiters, ${ }^{118}$ W. Erdmann, ${ }^{118}$ R. Horisberger, ${ }^{118}$ Q. Ingram, ${ }^{118}$ H. C. Kaestli, ${ }^{118}$

D. Kotlinski, ${ }^{118}$ U. Langenegger, ${ }^{118}$ T. Rohe, ${ }^{118}$ S. A. Wiederkehr, ${ }^{118}$ M. Backhaus, ${ }^{119}$ L. Bäni, ${ }^{119}$ P. Berger, ${ }^{119}$ N. Chernyavskaya, ${ }^{119}$ G. Dissertori, ${ }^{119}$ M. Dittmar, ${ }^{119}$ M. Donegà, ${ }^{119}$ C. Dorfer, ${ }^{119}$ T. A. Gómez Espinosa,${ }^{119}$ C. Grab,${ }^{119}$ D. Hits, ${ }^{119}$ T. Klijnsma, ${ }^{119}$ W. Lustermann, ${ }^{119}$ R. A. Manzoni, ${ }^{119}$ M. Marionneau, ${ }^{119}$ M. T. Meinhard ${ }^{119}$ F. Micheli, ${ }^{119}$ P. Musella, ${ }^{119}$ F. Nessi-Tedaldi ${ }^{119}$ J. Pata, ${ }^{119}$ F. Pauss,${ }^{119}$ G. Perrin,${ }^{119}$ L. Perrozzi, ${ }^{119}$ S. Pigazzini, ${ }^{119}$ M. Quittnat,${ }^{119}$ C. Reissel, ${ }^{119}$ D. Ruini, ${ }^{119}$ D. A. Sanz Becerra, ${ }^{119}$ M. Schönenberger, ${ }^{119}$ L. Shchutska, ${ }^{119}$ V. R. Tavolaro, ${ }^{119}$ K. Theofilatos, ${ }^{119}$ M. L. Vesterbacka Olsson, ${ }^{119}$ R. Wallny, ${ }^{119}$ D. H. Zhu, ${ }^{119}$ T. K. Aarrestad,${ }^{120}$ C. Amsler, ${ }^{120, y y}$ 
D. Brzhechko, ${ }^{120}$ M. F. Canelli, ${ }^{120}$ A. De Cosa, ${ }^{120}$ R. Del Burgo, ${ }^{120}$ S. Donato, ${ }^{120}$ C. Galloni, ${ }^{120}$ T. Hreus, ${ }^{120}$ B. Kilminster, ${ }^{120}$ S. Leontsinis, ${ }^{120}$ I. Neutelings, ${ }^{120}$ G. Rauco, ${ }^{120}$ P. Robmann, ${ }^{120}$ D. Salerno, ${ }^{120}$ K. Schweiger, ${ }^{120}$ C. Seitz, ${ }^{120}$ Y. Takahashi, ${ }^{120}$ A. Zucchetta, ${ }^{120}$ Y. H. Chang, ${ }^{121}$ K. y. Cheng, ${ }^{121}$ T. H. Doan, ${ }^{121}$ R. Khurana, ${ }^{121}$ C. M. Kuo, ${ }^{121}$ W. Lin,${ }^{121}$ A. Pozdnyakov, ${ }^{121}$ S. S. Yu, ${ }^{121}$ P. Chang, ${ }^{122}$ Y. Chao, ${ }^{122}$ K. F. Chen, ${ }^{122}$ P. H. Chen, ${ }^{122}$ W.-S. Hou, ${ }^{122}$ Arun Kumar, ${ }^{122}$ Y. F. Liu, ${ }^{122}$ R.-S. Lu, ${ }^{122}$ E. Paganis, ${ }^{122}$ A. Psallidas, ${ }^{122}$ A. Steen, ${ }^{122}$ B. Asavapibhop, ${ }^{123}$ N. Srimanobhas, ${ }^{123}$ N. Suwonjandee, ${ }^{123}$ M. N. Bakirci, ${ }^{124, z z}$ A. Bat, ${ }^{124}$ F. Boran, ${ }^{124}$ S. Cerci, ${ }^{124, \text { aaa }}$ S. Damarseckin, ${ }^{124}$ Z. S. Demiroglu, ${ }^{124}$ F. Dolek, ${ }^{124}$ C. Dozen, ${ }^{124}$ S. Girgis, ${ }^{124}$ G. Gokbulut, ${ }^{124}$ Y. Guler, ${ }^{124}$ E. Gurpinar, ${ }^{124}$ I. Hos, ${ }^{124, b b b}$ C. Isik, ${ }^{124}$ E. E. Kangal, ${ }^{124, c c c}$ O. Kara, ${ }^{124}$ A. Kayis Topaksu, ${ }^{124}$ U. Kiminsu, ${ }^{124}$ M. Oglakci, ${ }^{124}$ G. Onengut, ${ }^{124}$ K. Ozdemir, ${ }^{124, d d d}$ S. Ozturk, ${ }^{124, z z}$ D. Sunar Cerci, ${ }^{124, \text { aaa }}$ B. Tali, ${ }^{124, \text { aaa }}$ U. G. Tok, ${ }^{124}$ S. Turkcapar, ${ }^{124}$ I. S. Zorbakir, ${ }^{124}$ C. Zorbilmez, ${ }^{124}$ B. Isildak, ${ }^{125, \text { eee }}$ G. Karapinar, ${ }^{125, f f f}$ M. Yalvac, ${ }^{125}$ M. Zeyrek, ${ }^{125}$ I. O. Atakisi, ${ }^{126}$ E. Gülmez, ${ }^{126}$ M. Kaya, ${ }^{126, g g g}$ O. Kaya, ${ }^{126, \text { hhh }}$ S. Ozkorucuklu, ${ }^{126, \text { iii }}$ S. Tekten, ${ }^{126}$ E. A. Yetkin, ${ }^{126, j j j}$ M. N. Agaras, ${ }^{127}$ A. Cakir, ${ }^{127}$ K. Cankocak, ${ }^{127}$ Y. Komurcu, ${ }^{127}$ S. Sen,${ }^{127, k k k}$ B. Grynyov, ${ }^{128}$ L. Levchuk, ${ }^{129}$ F. Ball, ${ }^{130}$ L. Beck, ${ }^{130}$ J. J. Brooke, ${ }^{130}$ D. Burns, ${ }^{130}$ E. Clement, ${ }^{130}$ D. Cussans, ${ }^{130}$ O. Davignon, ${ }^{130}$ H. Flacher, ${ }^{130}$ J. Goldstein, ${ }^{130}$ G. P. Heath,${ }^{130}$ H. F. Heath, ${ }^{130}$ L. Kreczko, ${ }^{130}$ D. M. Newbold, ${ }^{130,111}$ S. Paramesvaran, ${ }^{130}$ B. Penning, ${ }^{130}$ T. Sakuma, ${ }^{130}$ D. Smith, ${ }^{130}$ V. J. Smith, ${ }^{130}$ J. Taylor, ${ }^{130}$ A. Titterton, ${ }^{130}$ K. W. Bell, ${ }^{131}$ A. Belyaev, ${ }^{131, \mathrm{mmm}}$ C. Brew, ${ }^{131}$ R. M. Brown, ${ }^{131}$ D. Cieri, ${ }^{131}$ D. J. A. Cockerill, ${ }^{131}$ J. A. Coughlan, ${ }^{131}$ K. Harder, ${ }^{131}$ S. Harper,${ }^{131}$ J. Linacre, ${ }^{131}$ E. Olaiya ${ }^{131}$ D. Petyt, ${ }^{131}$ C. H. Shepherd-Themistocleous, ${ }^{131}$ A. Thea ${ }^{131}$ I. R. Tomalin, ${ }^{131}$ T. Williams, ${ }^{131}$ W. J. Womersley, ${ }^{131}$ R. Bainbridge, ${ }^{132}$ P. Bloch, ${ }^{132}$ J. Borg, ${ }^{132}$ S. Breeze, ${ }^{132}$ O. Buchmuller, ${ }^{132}$ A. Bundock, ${ }^{132}$ D. Colling, ${ }^{132}$ P. Dauncey, ${ }^{132}$ G. Davies, ${ }^{132}$ M. Della Negra, ${ }^{132}$ R. Di Maria, ${ }^{132}$ Y. Haddad,${ }^{132}$ G. Hall, ${ }^{132}$ G. Iles, ${ }^{132}$ T. James, ${ }^{132}$ M. Komm, ${ }^{132}$ C. Laner, ${ }^{132}$ L. Lyons, ${ }^{132}$ A.-M. Magnan, ${ }^{132}$ S. Malik, ${ }^{132}$ A. Martelli, ${ }^{132}$ J. Nash,${ }^{132, n n n}$ A. Nikitenko, ${ }^{132, \mathrm{~h}}$ V. Palladino, ${ }^{132}$ M. Pesaresi, ${ }^{132}$ D. M. Raymond, ${ }^{132}$ A. Richards, ${ }^{132}$ A. Rose, ${ }^{132}$ E. Scott ${ }^{132}$ C. Seez, ${ }^{132}$ A. Shtipliyski, ${ }^{132}$ G. Singh, ${ }^{132}$ M. Stoye, ${ }^{132}$ T. Strebler, ${ }^{132}$ S. Summers ${ }^{132}$ A. Tapper, ${ }^{132}$ K. Uchida, ${ }^{132}$ T. Virdee,${ }^{132, \mathrm{r}}$ N. Wardle, ${ }^{132}$ D. Winterbottom, ${ }^{132}$ J. Wright, ${ }^{132}$ S. C. Zenz, ${ }^{132}$ J. E. Cole, ${ }^{133}$ P. R. Hobson, ${ }^{133}$ A. Khan, ${ }^{133}$ P. Kyberd, ${ }^{133}$ C. K. Mackay, ${ }^{133}$ A. Morton, ${ }^{133}$ I. D. Reid, ${ }^{133}$ L. Teodorescu, ${ }^{133}$ S. Zahid, ${ }^{133}$ K. Call, ${ }^{134}$ J. Dittmann, ${ }^{134}$ K. Hatakeyama, ${ }^{134}$ H. Liu, ${ }^{134}$ C. Madrid,${ }^{134}$ B. Mcmaster, ${ }^{134}$ N. Pastika,${ }^{134}$ C. Smith, ${ }^{134}$ R. Bartek, ${ }^{135}$ A. Dominguez, ${ }^{135}$ A. Buccilli, ${ }^{136}$ S. I. Cooper ${ }^{136}$ C. Henderson, ${ }^{136}$ P. Rumerio, ${ }^{136}$ C. West,${ }^{136}$ D. Arcaro, ${ }^{137}$ T. Bose, ${ }^{137}$ D. Gastler, ${ }^{137}$ D. Pinna, ${ }^{137}$ D. Rankin, ${ }^{137}$ C. Richardson, ${ }^{137}$ J. Rohlf, ${ }^{137}$ L. Sulak, ${ }^{137}$ D. Zou, ${ }^{137}$ G. Benelli, ${ }^{138}$ X. Coubez, ${ }^{138}$ D. Cutts, ${ }^{138}$ M. Hadley, ${ }^{138}$ J. Hakala, ${ }^{138}$ U. Heintz, ${ }^{138}$ J. M. Hogan, ${ }^{138,000}$ K. H. M. Kwok, ${ }^{138}$ E. Laird, ${ }^{138}$ G. Landsberg, ${ }^{138}$ J. Lee, ${ }^{138}$ Z. Mao, ${ }^{138}$ M. Narain, ${ }^{138}$ S. Sagir, ${ }^{138, p p p}$ R. Syarif, ${ }^{138}$ E. Usai, ${ }^{138}$ D. Yu, ${ }^{138}$ R. Band, ${ }^{139}$ C. Brainerd, ${ }^{139}$ R. Breedon, ${ }^{139}$ D. Burns, ${ }^{139}$ M. Calderon De La Barca Sanchez, ${ }^{139}$ M. Chertok, ${ }^{139}$ J. Conway, ${ }^{139}$ R. Conway, ${ }^{139}$ P. T. Cox, ${ }^{139}$ R. Erbacher, ${ }^{139}$ C. Flores, ${ }^{139}$ G. Funk, ${ }^{139}$ W. Ko, ${ }^{139}$ O. Kukral, ${ }^{139}$ R. Lander, ${ }^{139}$ M. Mulhearn, ${ }^{139}$ D. Pellett, ${ }^{139}$ J. Pilot, ${ }^{139}$ S. Shalhout, ${ }^{139}$ M. Shi, ${ }^{139}$ D. Stolp, ${ }^{139}$ D. Taylor,${ }^{139}$ K. Tos, ${ }^{139}$ M. Tripathi, ${ }^{139}$ Z. Wang, ${ }^{139}$ F. Zhang, ${ }^{139}$ M. Bachtis, ${ }^{140}$ C. Bravo, ${ }^{140}$ R. Cousins, ${ }^{140}$ A. Dasgupta, ${ }^{140}$ A. Florent, ${ }^{140}$ J. Hauser, ${ }^{140}$ M. Ignatenko, ${ }^{140}$ N. Mccoll, ${ }^{140}$ S. Regnard, ${ }^{140}$ D. Saltzberg, ${ }^{140}$ C. Schnaible, ${ }^{140}$ V. Valuev, ${ }^{140}$ E. Bouvier, ${ }^{141}$ K. Burt,${ }^{141}$ R. Clare,${ }^{141}$ J. W. Gary, ${ }^{141}$

S. M. A. Ghiasi Shirazi,${ }^{141}$ G. Hanson, ${ }^{141}$ G. Karapostoli, ${ }^{141}$ E. Kennedy, ${ }^{141}$ F. Lacroix, ${ }^{141}$ O. R. Long, ${ }^{141}$ M. Olmedo Negrete, ${ }^{141}$ M. I. Paneva, ${ }^{141}$ W. Si,${ }^{141}$ L. Wang, ${ }^{141}$ H. Wei ${ }^{141}$ S. Wimpenny, ${ }^{141}$ B. R. Yates,${ }^{141}$ J. G. Branson, ${ }^{142}$ P. Chang, ${ }^{142}$ S. Cittolin, ${ }^{142}$ M. Derdzinski, ${ }^{142}$ R. Gerosa, ${ }^{142}$ D. Gilbert, ${ }^{142}$ B. Hashemi, ${ }^{142}$ A. Holzner, ${ }^{142}$ D. Klein, ${ }^{142}$ G. Kole, ${ }^{142}$ V. Krutelyov, ${ }^{142}$ J. Letts, ${ }^{142}$ M. Masciovecchio, ${ }^{142}$ D. Olivito, ${ }^{142}$ S. Padhi, ${ }^{142}$ M. Pieri, ${ }^{142}$ M. Sani, ${ }^{142}$ V. Sharma, ${ }^{142}$ S. Simon, ${ }^{142}$ M. Tadel, ${ }^{142}$ A. Vartak, ${ }^{142}$ S. Wasserbaech, ${ }^{142, q q q}$ J. Wood, ${ }^{142}$ F. Würthwein, ${ }^{142}$ A. Yagil, ${ }^{142}$ G. Zevi Della Porta, ${ }^{142}$ N. Amin, ${ }^{143}$ R. Bhandari ${ }^{143}$ J. Bradmiller-Feld, ${ }^{143}$ C. Campagnari, ${ }^{143}$ M. Citron, ${ }^{143}$ A. Dishaw, ${ }^{143}$ V. Dutta, ${ }^{143}$ M. Franco Sevilla, ${ }^{143}$ L. Gouskos, ${ }^{143}$ R. Heller, ${ }^{143}$ J. Incandela, ${ }^{143}$ A. Ovcharova, ${ }^{143}$ H. Qu, ${ }^{143}$ J. Richman, ${ }^{143}$ D. Stuart, ${ }^{143}$ I. Suarez, ${ }^{143}$ S. Wang, ${ }^{143}$ J. Yoo, ${ }^{143}$ D. Anderson, ${ }^{144}$ A. Bornheim, ${ }^{144}$ J. M. Lawhorn, ${ }^{144}$ H. B. Newman, ${ }^{144}$ T. Q. Nguyen, ${ }^{144}$ M. Spiropulu, ${ }^{144}$ J. R. Vlimant, ${ }^{144}$ R. Wilkinson, ${ }^{144}$ S. Xie, ${ }^{144}$ Z. Zhang, ${ }^{144}$ R. Y. Zhu, ${ }^{144}$ M. B. Andrews, ${ }^{145}$ T. Ferguson, ${ }^{145}$ T. Mudholkar, ${ }^{145}$ M. Paulini, ${ }^{145}$ M. Sun, ${ }^{145}$ I. Vorobiev, ${ }^{145}$ M. Weinberg, ${ }^{145}$ J. P. Cumalat, ${ }^{146}$ W. T. Ford, ${ }^{146}$ F. Jensen, ${ }^{146}$ A. Johnson, ${ }^{146}$ M. Krohn, ${ }^{146}$ E. MacDonald, ${ }^{146}$ T. Mulholland, ${ }^{146}$ R. Patel, ${ }^{146}$ A. Perloff, ${ }^{146}$ K. Stenson, ${ }^{146}$ K. A. Ulmer, ${ }^{146}$ S. R. Wagner, ${ }^{146}$ J. Alexander,${ }^{147}$ J. Chaves, ${ }^{147}$ Y. Cheng, ${ }^{147}$ J. Chu, ${ }^{147}$ A. Datta, ${ }^{147}$ K. Mcdermott, ${ }^{147}$ N. Mirman, ${ }^{147}$ J. R. Patterson, ${ }^{147}$ D. Quach, ${ }^{147}$ A. Rinkevicius, ${ }^{147}$ A. Ryd ${ }^{147}$ L. Skinnari, ${ }^{147}$ L. Soffi, ${ }^{147}$ S. M. Tan, ${ }^{147}$ Z. Tao, ${ }^{147}$ J. Thom, ${ }^{147}$ J. Tucker,${ }^{147}$ P. Wittich,${ }^{147}$ M. Zientek, ${ }^{147}$ S. Abdullin,${ }^{148}$ M. Albrow, ${ }^{148}$ M. Alyari, ${ }^{148}$ G. Apollinari, ${ }^{148}$ A. Apresyan, ${ }^{148}$ A. Apyan, ${ }^{148}$ S. Banerjee, ${ }^{148}$ L. A. T. Bauerdick, ${ }^{148}$ A. Beretvas, ${ }^{148}$ J. Berryhill, ${ }^{148}$ P. C. Bhat, ${ }^{148}$ K. Burkett, ${ }^{148}$ J. N. Butler, ${ }^{148}$ A. Canepa, ${ }^{148}$ G. B. Cerati, ${ }^{148}$ H. W. K. Cheung, ${ }^{148}$ F. Chlebana, ${ }^{148}$ M. Cremonesi, ${ }^{148}$ 
J. Duarte, ${ }^{148}$ V. D. Elvira, ${ }^{148}$ J. Freeman, ${ }^{148}$ Z. Gecse, ${ }^{148}$ E. Gottschalk, ${ }^{148}$ L. Gray, ${ }^{148}$ D. Green, ${ }^{148}$ S. Grünendahl, ${ }^{148}$ O. Gutsche, ${ }^{148}$ J. Hanlon, ${ }^{148}$ R. M. Harris ${ }^{148}$ S. Hasegawa, ${ }^{148}$ J. Hirschauer ${ }^{148}$ Z. Hu, ${ }^{148}$ B. Jayatilaka, ${ }^{148}$ S. Jindariani, ${ }^{148}$ M. Johnson, ${ }^{148}$ U. Joshi, ${ }^{148}$ B. Klima, ${ }^{148}$ M. J. Kortelainen, ${ }^{148}$ B. Kreis, ${ }^{148}$ S. Lammel, ${ }^{148}$ D. Lincoln, ${ }^{148}$ R. Lipton, ${ }^{148}$ M. Liu, ${ }^{148}$ T. Liu, ${ }^{148}$ J. Lykken, ${ }^{148}$ K. Maeshima, ${ }^{148}$ J. M. Marraffino, ${ }^{148}$ D. Mason, ${ }^{148}$ P. McBride, ${ }^{148}$ P. Merkel, ${ }^{148}$ S. Mrenna, ${ }^{148}$ S. Nahn, ${ }^{148}$ V. O’Dell, ${ }^{148}$ K. Pedro, ${ }^{148}$ C. Pena, ${ }^{148}$ O. Prokofyev, ${ }^{148}$ G. Rakness, ${ }^{148}$ L. Ristori, ${ }^{148}$ A. Savoy-Navarro, ${ }^{148, \text { rrr }}$ B. Schneider, ${ }^{148}$ E. Sexton-Kennedy, ${ }^{148}$ A. Soha, ${ }^{148}$ W. J. Spalding, ${ }^{148}$ L. Spiegel, ${ }^{148}$ S. Stoynev, ${ }^{148}$ J. Strait, ${ }^{148}$ N. Strobbe, ${ }^{148}$ L. Taylor,${ }^{148}$ S. Tkaczyk, ${ }^{148}$ N. V. Tran, ${ }^{148}$ L. Uplegger, ${ }^{148}$ E. W. Vaandering, ${ }^{148}$ C. Vernieri, ${ }^{148}$ M. Verzocchi, ${ }^{148}$ R. Vidal, ${ }^{148}$ M. Wang, ${ }^{148}$ H. A. Weber, ${ }^{148}$ A. Whitbeck, ${ }^{148}$ D. Acosta, ${ }^{149}$ P. Avery, ${ }^{149}$ P. Bortignon, ${ }^{149}$ D. Bourilkov, ${ }^{149}$ A. Brinkerhoff, ${ }^{149}$ L. Cadamuro, ${ }^{149}$ A. Carnes,${ }^{149}$ M. Carver, ${ }^{149}$ D. Curry,${ }^{149}$ R. D. Field, ${ }^{149}$ S. V. Gleyzer, ${ }^{149}$ B. M. Joshi, ${ }^{149}$ J. Konigsberg, ${ }^{149}$ A. Korytov, ${ }^{149}$ K. H. Lo, ${ }^{149}$ P. Ma, ${ }^{149}$ K. Matchev, ${ }^{149}$ H. Mei, ${ }^{149}$ G. Mitselmakher, ${ }^{149}$ D. Rosenzweig, ${ }^{149}$ K. Shi, ${ }^{149}$ D. Sperka, ${ }^{149}$ J. Wang, ${ }^{149}$ S. Wang, ${ }^{149}$ X. Zuo,${ }^{149}$ Y. R. Joshi, ${ }^{150}$ S. Linn, ${ }^{150}$ A. Ackert, ${ }^{151}$ T. Adams, ${ }^{151}$ A. Askew, ${ }^{151}$ S. Hagopian, ${ }^{151}$ V. Hagopian, ${ }^{151}$ K. F. Johnson, ${ }^{151}$ T. Kolberg, ${ }^{151}$ G. Martinez,${ }^{151}$ T. Perry, ${ }^{151}$ H. Prosper, ${ }^{151}$ A. Saha, ${ }^{151}$ C. Schiber,${ }^{151}$ R. Yohay, ${ }^{151}$ M. M. Baarmand, ${ }^{152}$ V. Bhopatkar, ${ }^{152}$ S. Colafranceschi, ${ }^{152}$ M. Hohlmann, ${ }^{152}$ D. Noonan, ${ }^{152}$ M. Rahmani, ${ }^{152}$ T. Roy, ${ }^{152}$ F. Yumiceva, ${ }^{152}$ M. R. Adams, ${ }^{153}$ L. Apanasevich, ${ }^{153}$ D. Berry, ${ }^{153}$ R. R. Betts, ${ }^{153}$ R. Cavanaugh,${ }^{153}$ X. Chen, ${ }^{153}$ S. Dittmer, ${ }^{153}$ O. Evdokimov, ${ }^{153}$ C. E. Gerber, ${ }^{153}$ D. A. Hangal, ${ }^{153}$ D. J. Hofman, ${ }^{153}$ K. Jung, ${ }^{153}$ J. Kamin, ${ }^{153}$ C. Mills, ${ }^{153}$ I. D. Sandoval Gonzalez, ${ }^{153}$ M. B. Tonjes, ${ }^{153}$ H. Trauger, ${ }^{153}$ N. Varelas, ${ }^{153}$ H. Wang, ${ }^{153}$ X. Wang, ${ }^{153}$ Z. Wu,${ }^{153}$ J. Zhang, ${ }^{153}$ M. Alhusseini,${ }^{154}$ B. Bilki, ${ }^{154, \text { sss }}$ W. Clarida, ${ }^{154}$ K. Dilsiz, ${ }^{154, t t t}$ S. Durgut ${ }^{154}$ R. P. Gandrajula, ${ }^{154}$ M. Haytmyradov, ${ }^{154}$ V. Khristenko, ${ }^{154}$ J.-P. Merlo, ${ }^{154}$ A. Mestvirishvili, ${ }^{154}$ A. Moeller, ${ }^{154}$ J. Nachtman, ${ }^{154}$ H. Ogul, ${ }^{154, \text { uuu }}$ Y. Onel, ${ }^{154}$ F. Ozok, ${ }^{154, \text { vvv }}$ A. Penzo, ${ }^{154}$ C. Snyder,${ }^{154}$ E. Tiras,${ }^{154}$ J. Wetzel, ${ }^{154}$ B. Blumenfeld, ${ }^{155}$ A. Cocoros, ${ }^{155}$ N. Eminizer, ${ }^{155}$ D. Fehling, ${ }^{155}$ L. Feng, ${ }^{155}$ A. V. Gritsan, ${ }^{155}$ W. T. Hung, ${ }^{155}$ P. Maksimovic, ${ }^{155}$ J. Roskes, ${ }^{155}$ U. Sarica, ${ }^{155}$ M. Swartz, ${ }^{155}$ M. Xiao, ${ }^{155}$ C. You, ${ }^{155}$ A. Al-bataineh, ${ }^{156}$ P. Baringer, ${ }^{156}$ A. Bean, ${ }^{156}$ S. Boren, ${ }^{156}$ J. Bowen, ${ }^{156}$ A. Bylinkin, ${ }^{156}$ J. Castle, ${ }^{156}$ S. Khalil, ${ }^{156}$ A. Kropivnitskaya, ${ }^{156}$ D. Majumder, ${ }^{156}$ W. Mcbrayer, ${ }^{156}$ M. Murray, ${ }^{156}$ C. Rogan, ${ }^{156}$ S. Sanders, ${ }^{156}$ E. Schmitz, ${ }^{156}$ J. D. Tapia Takaki, ${ }^{156}$ Q. Wang, ${ }^{156}$ S. Duric, ${ }^{157}$ A. Ivanov, ${ }^{157}$ K. Kaadze, ${ }^{157}$ D. Kim, ${ }^{157}$ Y. Maravin, ${ }^{157}$ D. R. Mendis, ${ }^{157}$ T. Mitchell, ${ }^{157}$ A. Modak, ${ }^{157}$ A. Mohammadi, ${ }^{157}$ L. K. Saini, ${ }^{157}$ N. Skhirtladze, ${ }^{157}$ F. Rebassoo, ${ }^{158}$ D. Wright, ${ }^{158}$ A. Baden, ${ }^{159}$ O. Baron, ${ }^{159}$ A. Belloni, ${ }^{159}$ S. C. Eno, ${ }^{159}$ Y. Feng, ${ }^{159}$ C. Ferraioli, ${ }^{159}$ N. J. Hadley, ${ }^{159}$ S. Jabeen, ${ }^{159}$ G. Y. Jeng, ${ }^{159}$ R. G. Kellogg, ${ }^{159}$ J. Kunkle, ${ }^{159}$ A. C. Mignerey, ${ }^{159}$ S. Nabili, ${ }^{159}$ F. Ricci-Tam, ${ }^{159}$ Y. H. Shin, ${ }^{159}$ A. Skuja, ${ }^{159}$ S. C. Tonwar ${ }^{159}$ K. Wong, ${ }^{159}$ D. Abercrombie,${ }^{160}$ B. Allen, ${ }^{160}$ V. Azzolini, ${ }^{160}$ A. Baty, ${ }^{160}$ G. Bauer, ${ }^{160}$ R. Bi ${ }^{160}$ S. Brandt, ${ }^{160}$ W. Busza, ${ }^{160}$ I. A. Cali, ${ }^{160}$ M. D' Alfonso, ${ }^{160}$ Z. Demiragli, ${ }^{160}$ G. Gomez Ceballos, ${ }^{160}$ M. Goncharov, ${ }^{160}$ P. Harris, ${ }^{160}$ D. Hsu, ${ }^{160}$ M. Hu, ${ }^{160}$ Y. Iiyama, ${ }^{160}$ G. M. Innocenti, ${ }^{160}$ M. Klute, ${ }^{160}$ D. Kovalskyi, ${ }^{160}$ Y.-J. Lee, ${ }^{160}$ P. D. Luckey, ${ }^{160}$ B. Maier, ${ }^{160}$ A. C. Marini, ${ }^{160}$ C. Mcginn, ${ }^{160}$ C. Mironov, ${ }^{160}$ S. Narayanan, ${ }^{160}$ X. Niu, ${ }^{160}$ C. Paus, ${ }^{160}$ C. Roland, ${ }^{160}$ G. Roland, ${ }^{160}$ G. S. F. Stephans, ${ }^{160}$ K. Sumorok, ${ }^{160}$ K. Tatar, ${ }^{160}$ D. Velicanu, ${ }^{160}$ J. Wang, ${ }_{160}^{160}$ T. W. Wang, ${ }^{160}$ B. Wyslouch, ${ }^{160}$ S. Zhaozhong, ${ }^{160}$ A. C. Benvenuti, ${ }^{161, a}$ R. M. Chatterjee, ${ }^{161}$ A. Evans, ${ }^{161}$ P. Hansen, ${ }^{161}$ J. Hiltbrand, ${ }^{161}$ Sh. Jain, ${ }^{161}$ S. Kalafut, ${ }^{161}$ Y. Kubota, ${ }^{161}$ Z. Lesko, ${ }^{161}$ J. Mans, ${ }^{161}$ N. Ruckstuhl, ${ }^{161}$ R. Rusack, ${ }^{161}$ M. A. Wadud,${ }^{161}$ J. G. Acosta, ${ }^{162}$ S. Oliveros, ${ }^{162}$ E. Avdeeva, ${ }^{163}$ K. Bloom, ${ }^{163}$ D. R. Claes, ${ }^{163}$ C. Fangmeier, ${ }^{163}$ F. Golf, ${ }^{163}$ R. Gonzalez Suarez, ${ }^{163}$ R. Kamalieddin, ${ }^{163}$ I. Kravchenko, ${ }^{163}$ J. Monroy, ${ }^{163}$ J. E. Siado, ${ }^{163}$ G. R. Snow, ${ }^{163}$ B. Stieger, ${ }^{163}$ A. Godshalk, ${ }^{164}$ C. Harrington, ${ }^{164}$ I. Iashvili, ${ }^{164}$ A. Kharchilava, ${ }^{164}$ C. Mclean, ${ }^{164}$ D. Nguyen, ${ }^{164}$ A. Parker, ${ }^{164}$ S. Rappoccio, ${ }^{164}$ B. Roozbahani, ${ }^{164}$ G. Alverson, ${ }^{165}$ E. Barberis, ${ }^{165}$ C. Freer, ${ }^{165}$ A. Hortiangtham ${ }^{165}$ D. M. Morse, ${ }^{165}$ T. Orimoto, ${ }^{165}$ R. Teixeira De Lima, ${ }^{165}$ T. Wamorkar, ${ }^{165}$ B. Wang, ${ }^{165}$ A. Wisecarver, ${ }^{165} \mathrm{D}$. Wood, ${ }^{165}$ S. Bhattacharya, ${ }^{166}$ O. Charaf, ${ }^{166}$ K. A. Hahn, ${ }^{166}$ N. Mucia, ${ }^{166}$ N. Odell, ${ }^{166}$ M. H. Schmitt, ${ }^{166}$ K. Sung, ${ }^{166}$ M. Trovato, ${ }^{166}$ M. Velasco, ${ }^{166}$ R. Bucci, ${ }^{167}$ N. Dev ${ }^{167}$ M. Hildreth, ${ }^{167}$ K. Hurtado Anampa,${ }^{167}$ C. Jessop, ${ }^{167}$ D. J. Karmgard, ${ }^{167}$ N. Kellams, ${ }^{167}$ K. Lannon, ${ }^{167}$ W. Li, ${ }^{167}$ N. Loukas, ${ }^{167}$ N. Marinelli, ${ }^{167}$ F. Meng, ${ }^{167}$ C. Mueller, ${ }^{167}$ Y. Musienko, ${ }^{167, k \mathrm{kk}}$ M. Planer, ${ }^{167}$ A. Reinsvold, ${ }^{167}$ R. Ruchti, ${ }^{167}$ P. Siddireddy, ${ }^{167}$ G. Smith, ${ }^{167}$ S. Taroni, ${ }^{167}$ M. Wayne, ${ }^{167}$ A. Wightman, ${ }^{167}$ M. Wolf, ${ }^{167}$ A. Woodard, ${ }^{167}$ J. Alimena, ${ }^{168}$ L. Antonelli, ${ }^{168}$ B. Bylsma, ${ }^{168}$ L. S. Durkin, ${ }^{168}$ S. Flowers, ${ }^{168}$ B. Francis, ${ }^{168}$ A. Hart, ${ }^{168}$ C. Hill, ${ }^{168}$ W. Ji, ${ }^{168}$ T. Y. Ling, ${ }^{168}$ W. Luo, ${ }^{168}$ B. L. Winer, ${ }^{168}$ S. Cooperstein, ${ }^{169}$ P. Elmer, ${ }^{169}$ J. Hardenbrook, ${ }^{169}$ S. Higginbotham, ${ }^{169}$ A. Kalogeropoulos ${ }^{169}$ D. Lange, ${ }^{169}$ M. T. Lucchini, ${ }^{169}$ J. Luo, ${ }^{169}$ D. Marlow, ${ }^{169}$ K. Mei, ${ }^{169}$ I. Ojalvo, ${ }^{169}$ J. Olsen, ${ }^{169}$ C. Palmer, ${ }^{169}$ P. Piroué, ${ }^{169}$ J. Salfeld-Nebgen, ${ }^{169}$ D. Stickland, ${ }^{169}$ C. Tully, ${ }^{169}$ S. Malik, ${ }^{170}$ S. Norberg, ${ }^{170}$ A. Barker, ${ }^{171}$ V. E. Barnes, ${ }^{171}$ S. Das, ${ }^{171}$ L. Gutay, ${ }^{171}$ M. Jones, ${ }^{171}$ A. W. Jung, ${ }^{171}$ A. Khatiwada, ${ }^{171}$ B. Mahakud, ${ }^{171}$ D. H. Miller ${ }^{171}$ N. Neumeister, ${ }^{171}$ C. C. Peng, ${ }^{171}$ S. Piperov,${ }^{171}$ H. Qiu, ${ }^{171}$ J. F. Schulte,${ }^{171}$ J. Sun, ${ }^{171}$ F. Wang, ${ }^{171}$ R. Xiao, ${ }^{171}$ W. Xie, ${ }^{171}$ T. Cheng, ${ }^{172}$ J. Dolen, ${ }^{172}$ N. Parashar, ${ }^{172}$ Z. Chen, ${ }^{173}$ K. M. Ecklund, ${ }^{173}$ S. Freed, ${ }^{173}$ F. J. M. Geurts, ${ }^{173}$ 
M. Kilpatrick, ${ }^{173}$ W. Li, ${ }^{173}$ B.P. Padley, ${ }^{173}$ R. Redjimi, ${ }^{173}$ J. Roberts, ${ }^{173}$ J. Rorie, ${ }^{173}$ W. Shi, ${ }^{173}$ Z. Tu, ${ }^{173}$ J. Zabel, ${ }^{173}$ A. Zhang, ${ }^{173}$ A. Bodek, ${ }^{174}$ P. de Barbaro, ${ }^{174}$ R. Demina, ${ }^{174}$ Y. t. Duh, ${ }^{174}$ J. L. Dulemba, ${ }^{174}$ C. Fallon, ${ }^{174}$ T. Ferbel, ${ }^{174}$ M. Galanti, ${ }^{174}$ A. Garcia-Bellido, ${ }^{174}$ J. Han, ${ }^{174}$ O. Hindrichs, ${ }^{174}$ A. Khukhunaishvili, ${ }^{174}$ P. Tan, ${ }^{174}$ R. Taus, ${ }^{174}$ A. Agapitos, ${ }^{175}$ J. P. Chou, ${ }^{175}$ Y. Gershtein, ${ }^{175}$ E. Halkiadakis, ${ }^{175}$ M. Heindl, ${ }^{175}$ E. Hughes, ${ }^{175}$ S. Kaplan, ${ }^{175}$ R. Kunnawalkam Elayavalli, ${ }^{175}$ S. Kyriacou, ${ }^{175}$ A. Lath, ${ }^{175}$ R. Montalvo, ${ }^{175}$ K. Nash, ${ }^{175}$ M. Osherson, ${ }^{175}$ H. Saka, ${ }^{175}$ S. Salur, ${ }^{175}$ S. Schnetzer, ${ }^{175}$ D. Sheffield, ${ }^{175}$ S. Somalwar, ${ }^{175}$ R. Stone, ${ }^{175}$ S. Thomas, ${ }^{175}$ P. Thomassen, ${ }^{175}$ M. Walker, ${ }^{175}$ A. G. Delannoy, ${ }^{176}$ J. Heideman, ${ }^{176}$ G. Riley, ${ }^{176}$ S. Spanier,${ }^{176}$ O. Bouhali, ${ }^{177, \text { www }}$ A. Celik, ${ }^{177}$ M. Dalchenko, ${ }^{177}$ M. De Mattia, ${ }^{177}$ A. Delgado, ${ }^{177}$ S. Dildick, ${ }^{177}$ R. Eusebi,${ }^{177}$ J. Gilmore ${ }^{177}$ T. Huang, ${ }^{177}$ T. Kamon, ${ }^{177, x x x}$ S. Luo, ${ }^{177}$ R. Mueller, ${ }^{177}$ D. Overton, ${ }^{177}$ L. Perniè,${ }^{177}$ D. Rathjens, ${ }^{177}$ A. Safonov, ${ }^{177}$ N. Akchurin, ${ }^{178}$ J. Damgov, ${ }^{178}$ F. De Guio, ${ }^{178}$ P. R. Dudero, ${ }^{178}$ S. Kunori, ${ }^{178}$ K. Lamichhane, ${ }^{178}$ S. W. Lee, ${ }^{178}$ T. Mengke, ${ }^{178}$ S. Muthumuni, ${ }^{178}$ T. Peltola, ${ }^{178}$ S. Undleeb,${ }^{178}$ I. Volobouev, ${ }^{178}$ Z. Wang, ${ }^{178}$ S. Greene, ${ }^{179}$ A. Gurrola, ${ }^{179}$ R. Janjam, ${ }^{179}$ W. Johns, ${ }^{179}$ C. Maguire, ${ }^{179}$ A. Melo, ${ }^{179} \mathrm{H} . \mathrm{Ni},{ }^{179} \mathrm{~K}$. Padeken, ${ }^{179}$ J. D. Ruiz Alvarez, ${ }^{179}$ P. Sheldon, ${ }^{179}$ S. Tuo, ${ }^{179}$ J. Velkovska, ${ }^{179}$ M. Verweij, ${ }^{179}$ Q. Xu, ${ }^{179}$ M. W. Arenton, ${ }^{180}$ P. Barria, ${ }^{180}$ B. Cox ${ }^{180}$ R. Hirosky, ${ }^{180}$ M. Joyce, ${ }^{180}$ A. Ledovskoy, ${ }^{180}$ H. Li, ${ }^{180}$ C. Neu, ${ }^{180}$ T. Sinthuprasith, ${ }^{180}$ Y. Wang, ${ }^{180}$ E. Wolfe, ${ }^{180}$ F. Xia, ${ }^{180}$ R. Harr, ${ }^{181}$ P. E. Karchin, ${ }^{181}$ N. Poudyal, ${ }^{181}$ J. Sturdy, ${ }^{181}$ P. Thapa ${ }^{181}$ S. Zaleski,${ }^{181}$ M. Brodski, ${ }^{182}$ J. Buchanan, ${ }^{182}$ C. Caillol, ${ }^{182}$ D. Carlsmith, ${ }^{182}$ S. Dasu, ${ }^{182}$ L. Dodd ${ }^{182}$ B. Gomber, ${ }^{182}$ M. Grothe, ${ }^{182}$ M. Herndon, ${ }^{182}$ A. Hervé, ${ }^{182}$ U. Hussain, ${ }^{182}$ P. Klabbers, ${ }^{182}$ A. Lanaro, ${ }^{182}$ K. Long, ${ }^{182}$ R. Loveless, ${ }^{182}$ T. Ruggles, ${ }^{182}$ A. Savin, ${ }^{182}$ V. Sharma,${ }^{182}$ N. Smith, ${ }^{182}$ W. H. Smith, ${ }^{182}$ and N. Woods ${ }^{182}$

\title{
(CMS Collaboration)
}

\author{
${ }^{1}$ Yerevan Physics Institute, Yerevan, Armenia \\ ${ }^{2}$ Institut für Hochenergiephysik, Wien, Austria \\ ${ }^{3}$ Institute for Nuclear Problems, Minsk, Belarus \\ ${ }^{4}$ Universiteit Antwerpen, Antwerpen, Belgium \\ ${ }^{5}$ Vrije Universiteit Brussel, Brussel, Belgium \\ ${ }^{6}$ Université Libre de Bruxelles, Bruxelles, Belgium \\ ${ }^{7}$ Ghent University, Ghent, Belgium \\ ${ }^{8}$ Université Catholique de Louvain, Louvain-la-Neuve, Belgium \\ ${ }^{9}$ Centro Brasileiro de Pesquisas Fisicas, Rio de Janeiro, Brazil \\ ${ }^{10}$ Universidade do Estado do Rio de Janeiro, Rio de Janeiro, Brazil \\ ${ }^{11 a}$ Universidade Estadual Paulista, São Paulo, Brazil \\ ${ }^{11 \mathrm{~b}}$ Universidade Federal do ABC, São Paulo, Brazil \\ ${ }^{12}$ Institute for Nuclear Research and Nuclear Energy, Bulgarian Academy of Sciences, Sofia, Bulgaria \\ ${ }^{13}$ University of Sofia, Sofia, Bulgaria \\ ${ }^{14}$ Beihang University, Beijing, China \\ ${ }^{15}$ Institute of High Energy Physics, Beijing, China \\ ${ }^{16}$ State Key Laboratory of Nuclear Physics and Technology, Peking University, Beijing, China \\ ${ }^{17}$ Tsinghua University, Beijing, China \\ ${ }^{18}$ Universidad de Los Andes, Bogota, Colombia \\ ${ }^{19}$ University of Split, Faculty of Electrical Engineering, Mechanical Engineering and Naval Architecture, Split, Croatia \\ ${ }^{20}$ University of Split, Faculty of Science, Split, Croatia \\ ${ }^{21}$ Institute Rudjer Boskovic, Zagreb, Croatia \\ ${ }^{22}$ University of Cyprus, Nicosia, Cyprus \\ ${ }^{23}$ Charles University, Prague, Czech Republic \\ ${ }^{24}$ Escuela Politecnica Nacional, Quito, Ecuador \\ ${ }^{25}$ Universidad San Francisco de Quito, Quito, Ecuador \\ ${ }^{26}$ Academy of Scientific Research and Technology of the Arab Republic of Egypt, \\ Egyptian Network of High Energy Physics, Cairo, Egypt \\ ${ }^{27}$ National Institute of Chemical Physics and Biophysics, Tallinn, Estonia \\ ${ }^{28}$ Department of Physics, University of Helsinki, Helsinki, Finland \\ ${ }^{29}$ Helsinki Institute of Physics, Helsinki, Finland \\ ${ }^{30}$ Lappeenranta University of Technology, Lappeenranta, Finland \\ ${ }^{31}$ IRFU, CEA, Université Paris-Saclay, Gif-sur-Yvette, France \\ ${ }^{32}$ Laboratoire Leprince-Ringuet, Ecole polytechnique, CNRS/IN2P3, Université Paris-Saclay, Palaiseau, France \\ ${ }^{33}$ Université de Strasbourg, CNRS, IPHC UMR 7178, Strasbourg, France
}


${ }^{34}$ Centre de Calcul de l'Institut National de Physique Nucleaire et de Physique des Particules, CNRS/IN2P3, Villeurbanne, France

${ }^{35}$ Université de Lyon, Université Claude Bernard Lyon 1, CNRS-IN2P3, Institut de Physique Nucléaire de Lyon, Villeurbanne, France

${ }^{36}$ Georgian Technical University, Tbilisi, Georgia

${ }^{37}$ Tbilisi State University, Tbilisi, Georgia

${ }^{38}$ RWTH Aachen University, I. Physikalisches Institut, Aachen, Germany

${ }^{39}$ RWTH Aachen University, III. Physikalisches Institut A, Aachen, Germany

${ }^{40}$ RWTH Aachen University, III. Physikalisches Institut B, Aachen, Germany

${ }^{41}$ Deutsches Elektronen-Synchrotron, Hamburg, Germany

${ }^{42}$ University of Hamburg, Hamburg, Germany

${ }^{43}$ Karlsruher Institut fuer Technologie, Karlsruhe, Germany

${ }^{44}$ Institute of Nuclear and Particle Physics (INPP), NCSR Demokritos, Aghia Paraskevi, Greece

${ }^{45}$ National and Kapodistrian University of Athens, Athens, Greece

${ }^{46}$ National Technical University of Athens, Athens, Greece

${ }^{47}$ University of Ioánnina, Ioánnina, Greece

${ }^{48}$ MTA-ELTE Lendület CMS Particle and Nuclear Physics Group, Eötvös Loránd University, Budapest, Hungary

${ }^{49}$ Wigner Research Centre for Physics, Budapest, Hungary

${ }^{50}$ Institute of Nuclear Research ATOMKI, Debrecen, Hungary

${ }^{51}$ Institute of Physics, University of Debrecen, Debrecen, Hungary

${ }^{52}$ Indian Institute of Science (IISc), Bangalore, India

${ }^{53}$ National Institute of Science Education and Research, HBNI, Bhubaneswar, India

${ }^{54}$ Panjab University, Chandigarh, India

${ }^{55}$ University of Delhi, Delhi, India

${ }^{56}$ Saha Institute of Nuclear Physics, HBNI, Kolkata,India

${ }^{57}$ Indian Institute of Technology Madras, Madras, India

${ }^{58}$ Bhabha Atomic Research Centre, Mumbai, India

${ }^{59}$ Tata Institute of Fundamental Research-A, Mumbai, India

${ }^{60}$ Tata Institute of Fundamental Research-B, Mumbai, India

${ }^{61}$ Indian Institute of Science Education and Research (IISER), Pune, India

${ }^{62}$ Institute for Research in Fundamental Sciences (IPM), Tehran, Iran

${ }^{63}$ University College Dublin, Dublin, Ireland

${ }^{64 a}$ INFN Sezione di Bari, Bari, Italy

${ }^{64 \mathrm{~b}}$ Università di Bari, Bari, Italy

${ }^{64 \mathrm{c}}$ Politecnico di Bari, Bari, Italy

${ }^{65 \mathrm{a}}$ INFN Sezione di Bologna, Bologna, Italy

${ }^{65 \mathrm{~b}}$ Università di Bologna, Bologna, Italy

${ }^{66 \mathrm{a}}$ INFN Sezione di Catania, Catania, Italy

${ }^{66 \mathrm{~b}}$ Università di Catania, Catania, Italy

${ }^{67 \mathrm{a}}$ INFN Sezione di Firenze, Firenze, Italy

${ }^{67 \mathrm{~b}}$ Università di Firenze, Firenze, Italy

${ }^{68}$ INFN Laboratori Nazionali di Frascati, Frascati, Italy

${ }^{69 a}$ INFN Sezione di Genova, Genova, Italy

${ }^{69 \mathrm{~b}}$ Università di Genova, Genova, Italy

${ }^{70 a}$ INFN Sezione di Milano-Bicocca, Milano, Italy

${ }^{70 \mathrm{~b}}$ Università di Milano-Bicocca, Milano, Italy

${ }^{71 a}$ INFN Sezione di Napoli, Napoli, Italy

${ }^{71 \mathrm{~b}}$ Università di Napoli 'Federico II', Napoli, Italy

${ }^{71 \mathrm{c}}$ Università della Basilicata, Potenza, Italy

${ }^{71 \mathrm{~d}}$ Università G. Marconi, Roma, Italy

${ }^{72 a}$ INFN Sezione di Padova, Padova, Italy

${ }^{72 \mathrm{~b}}$ Università di Padova, Padova, Italy

${ }^{72 \mathrm{c}}$ Università di Trento, Trento, Italy

${ }^{73 a}$ INFN Sezione di Pavia

${ }^{73 \mathrm{~b}}$ Università di Pavia

${ }^{74 a}$ INFN Sezione di Perugia, Perugia, Italy

${ }^{74 \mathrm{~b}}$ Università di Perugia, Perugia, Italy

${ }^{75 a}$ INFN Sezione di Pisa, Pisa, Italy

${ }^{75 b}$ Università di Pisa, Pisa, Italy

${ }^{75 c}$ Scuola Normale Superiore di Pisa, Pisa, Italy

${ }^{76 a}$ INFN Sezione di Roma, Rome, Italy

${ }^{76 \mathrm{~b}}$ Sapienza Università di Roma, Rome, Italy 
${ }^{77 a}$ INFN Sezione di Torino, Torino, Italy

${ }^{77 b}$ Università di Torino, Torino, Italy

${ }^{77 \mathrm{c}}$ Università del Piemonte Orientale, Novara, Italy

${ }^{78 a}$ INFN Sezione di Trieste, Trieste, Italy

${ }^{78 b}$ Università di Trieste, Trieste, Italy

${ }^{79}$ Kyungpook National University, Daegu, Korea

${ }^{80}$ Chonnam National University, Institute for Universe and Elementary Particles, Kwangju, Korea

${ }^{81}$ Hanyang University, Seoul, Korea

${ }^{82}$ Korea University, Seoul, Korea

${ }^{83}$ Sejong University, Seoul, Korea

${ }^{84}$ Seoul National University, Seoul, Korea

${ }^{85}$ University of Seoul, Seoul, Korea

${ }^{86}$ Sungkyunkwan University, Suwon, Korea

${ }^{87}$ Vilnius University, Vilnius, Lithuania

${ }^{88}$ National Centre for Particle Physics, Universiti Malaya, Kuala Lumpur, Malaysia

${ }^{89}$ Universidad de Sonora (UNISON), Hermosillo, Mexico

${ }^{90}$ Centro de Investigacion y de Estudios Avanzados del IPN, Mexico City, Mexico

${ }^{91}$ Universidad Iberoamericana, Mexico City, Mexico

${ }^{92}$ Benemerita Universidad Autonoma de Puebla, Puebla, Mexico

${ }^{93}$ Universidad Autónoma de San Luis Potosí, San Luis Potosí, Mexico

${ }^{94}$ University of Auckland, Auckland, New Zealand

${ }^{95}$ University of Canterbury, Christchurch, New Zealand

${ }^{96}$ National Centre for Physics, Quaid-I-Azam University, Islamabad, Pakistan

${ }^{97}$ National Centre for Nuclear Research, Swierk, Poland

${ }^{98}$ Institute of Experimental Physics, Faculty of Physics, University of Warsaw, Warsaw, Poland

${ }^{99}$ Laboratório de Instrumentação e Física Experimental de Partículas, Lisboa, Portugal

${ }^{100}$ Joint Institute for Nuclear Research, Dubna, Russia

${ }^{101}$ Petersburg Nuclear Physics Institute, Gatchina (St. Petersburg), Russia

${ }^{102}$ Institute for Nuclear Research, Moscow, Russia

${ }^{103}$ Institute for Theoretical and Experimental Physics, Moscow, Russia

${ }^{104}$ Moscow Institute of Physics and Technology, Moscow, Russia

${ }^{105}$ National Research Nuclear University 'Moscow Engineering Physics Institute' (MEPhI), Moscow, Russia

${ }^{106}$ P.N. Lebedev Physical Institute, Moscow, Russia

${ }^{107}$ Skobeltsyn Institute of Nuclear Physics, Lomonosov Moscow State University, Moscow, Russia

${ }^{108}$ Novosibirsk State University (NSU), Novosibirsk, Russia

${ }^{109}$ Institute for High Energy Physics of National Research Centre 'Kurchatov Institute', Protvino, Russia

${ }^{110}$ National Research Tomsk Polytechnic University, Tomsk, Russia

${ }^{111}$ University of Belgrade, Faculty of Physics and Vinca Institute of Nuclear Sciences, Belgrade, Serbia

${ }^{112}$ Centro de Investigaciones Energéticas Medioambientales y Tecnológicas (CIEMAT), Madrid, Spain

${ }^{113}$ Universidad Autónoma de Madrid, Madrid, Spain

${ }^{114}$ Universidad de Oviedo, Oviedo, Spain

${ }^{115}$ Instituto de Física de Cantabria (IFCA), CSIC-Universidad de Cantabria, Santander, Spain

${ }^{116}$ University of Ruhuna, Department of Physics, Matara, Sri Lanka

${ }^{117}$ CERN, European Organization for Nuclear Research, Geneva, Switzerland

${ }^{118}$ Paul Scherrer Institut, Villigen, Switzerland

${ }^{119}$ ETH Zurich-Institute for Particle Physics and Astrophysics (IPA), Zurich, Switzerland

${ }^{120}$ Universität Zürich, Zurich, Switzerland

${ }^{121}$ National Central University, Chung-Li, Taiwan

${ }^{122}$ National Taiwan University (NTU), Taipei, Taiwan

${ }^{123}$ Chulalongkorn University, Faculty of Science, Department of Physics, Bangkok, Thailand

${ }^{124}$ Cukurova University, Physics Department, Science and Art Faculty, Adana, Turkey

${ }^{125}$ Middle East Technical University, Physics Department, Ankara, Turkey

${ }^{126}$ Bogazici University, Istanbul, Turkey

${ }^{127}$ Istanbul Technical University, Istanbul, Turkey

${ }^{128}$ Institute for Scintillation Materials of National Academy of Science of Ukraine, Kharkov, Ukraine

${ }^{129}$ National Scientific Center, Kharkov Institute of Physics and Technology, Kharkov, Ukraine

${ }^{130}$ University of Bristol, Bristol, United Kingdom

${ }^{131}$ Rutherford Appleton Laboratory, Didcot, United Kingdom

${ }^{132}$ Imperial College, London, United Kingdom

${ }^{133}$ Brunel University, Uxbridge, United Kingdom 


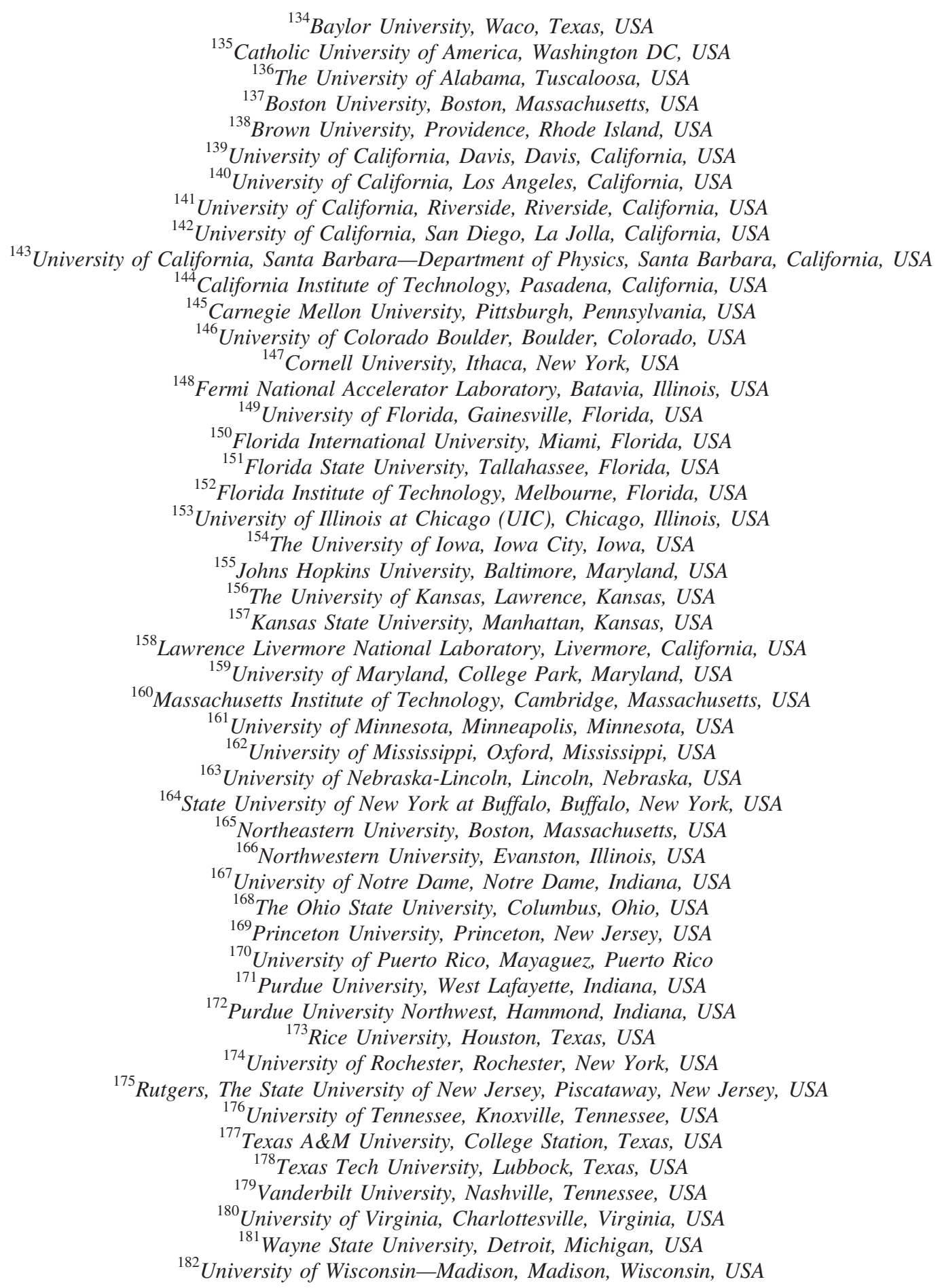

${ }^{\mathrm{a}}$ Deceased.

${ }^{\mathrm{b}}$ Also at Vienna University of Technology, Vienna, Austria.

${ }^{c}$ Also at IRFU, CEA, Université Paris-Saclay, Gif-sur-Yvette, France.

${ }^{\mathrm{d}}$ Also at Universidade Estadual de Campinas, Campinas, Brazil.

${ }^{\mathrm{e}}$ Also at Federal University of Rio Grande do Sul, Porto Alegre, Brazil.

${ }^{\mathrm{f}}$ Also at Université Libre de Bruxelles, Bruxelles, Belgium.

${ }^{\mathrm{g}}$ Also at University of Chinese Academy of Sciences.

${ }^{\mathrm{h}}$ Also at Institute for Theoretical and Experimental Physics, Moscow, Russia.

${ }^{\mathrm{i}}$ Also at Joint Institute for Nuclear Research, Dubna, Russia.

${ }^{\mathrm{j}}$ Also at Helwan University, Cairo, Egypt. 


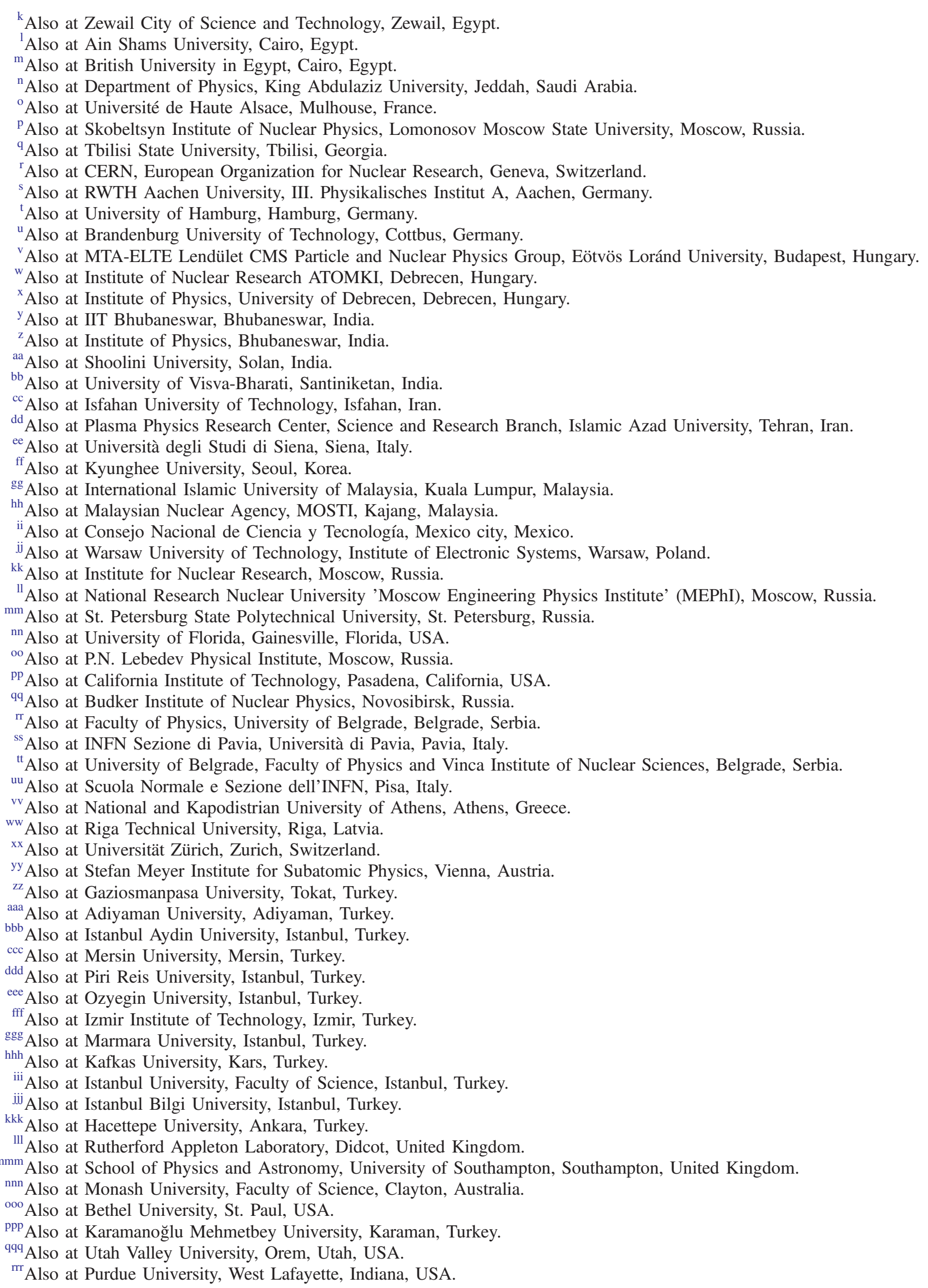




\footnotetext{
${ }^{\text {sss }}$ Also at Beykent University, Istanbul, Turkey.

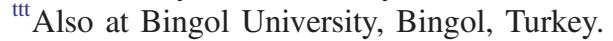

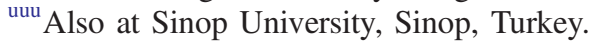

${ }^{\mathrm{vvv}}$ Also at Mimar Sinan University, Istanbul, Istanbul, Turkey.

${ }^{\text {www }}$ Also at Texas A\&M University at Qatar, Doha, Qatar.

${ }^{\mathrm{xxx}}$ Also at Kyungpook National University, Daegu, Korea.
} 